Benjamin Gittel

\title{
Lässt sich literarischer Wandel erklären? Struktur, Gültigkeitsbedingungen und Reichweite verschiedener Erklärungstypen in der Literaturgeschichtsschreibung
}

DOI 10.1515/jlt-2016-0012

Abstract: Although literary critics continue to make programmatic claims about not only describing but also explaining literary change, and numerous textbooks and individual studies in literary history insinuate or claim to explain literary change, explanations of literary change are as of yet insufficiently reflected in the field's methodology. Is it at all possible to provide explanations in literary history, where no strict laws have been discovered yet? If yes, what do these explanations look like and in which circumstances are they valid? Understanding literary change as the variance in a specific genre's instantiation over time, this paper works from the point of departure of explanatory pluralism, the assumption that scientific explanations are to a certain degree discipline-specific and that various different types of explanations exist. The paper aims at an interpenetration of theory and practice and therefore analyzes different types of explanations through a concrete example of literary change. In particular, it focuses on the boom of fictional essay writing that occurred during the first third of the 20th century in German-speaking countries, thus analyzing the two trends of the fictionalization of the essay and the insertion of essayistic passages into fictional texts (e. g., the essayistic novel). The paper examines causal, statistical, intentional, functional, teleological and structural explanations for this literary change.

Causal explanations, it is argued, cannot be employed as long as no general laws for literary change have been identified. However, it is possible to identify certain causal factors for literary change through the interplay of biographic and intertextual studies, which can be further validated by statistical approaches. Intentional explanations of literary change can generally be created through the time-consuming process of collecting explanations for the writing of single works, but they face the problem that author's intentions, as reported in self-commentaries or poetological texts, are often too unspecific or too specific for the work being explained. Functional explanations face two difficulties. First, literature

Kontaktperson: Benjamin Gittel: Humboldt-Universität zu Berlin, Institut für deutsche Literatur, E-Mail: benjamin.gittel@hu-berlin.de 
rarely solves social problems, and therefore the benefit that functional explanations presuppose can usually only consist in the thematization of social problems. Second, the causal feedback mechanism that underlies functional explanations presupposes a mechanism for social selection amongst works of literature that promotes works that have social benefit. However, only a very idealized literature market could provide for social selection along these lines. Teleological explanations, which ascribe inherent development trends to genres, are not only dubious from the perspective of the philosophy of science, but fail to explain why these trends manifest themselves in specific historical situations. Structural explanations identify underlying >deep structures of text corpora that might correspond to social or ideational structures. These explanations, however, are also questionable, because they usually don't provide information about the causal mechanisms that may lead to this correspondence.

Two consequences, this paper argues, can be drawn from the analysis of various types of explanation of literary change. Concluding that full-fledged explanations of literary change are either very time-consuming and laborious (statistical and additive intentional explanations), or only employable under specific conditions and idealizing background assumptions (functional explanations), it suggests the following. First, literary scholars could revise their practice of answering swhy questions in literary history and abstain from explaining literary change except in those cases when they elaborated full-fledged explanations. Second, alternatively, they could continue their existing practice but refrain from describing it as sexplaining literary change. Instead, they could describe their activity in less demanding terms, e. g. as sthe search for overarching narratives, which nevertheless is of value in terms of didactics, knowledge synthesis, or the reduction of complexity.

Schlagworte: explanatorischer Pluralismus, funktionale Erklärung, Funktion, literarische Evolution, Literaturgeschichte

\section{Einleitung}

Es sei »die vorrangige Aufgabe einer Literaturgeschichte [...], die Ursachen, Erscheinungsarten und Prozeduren des literarischen Wandels darzustellen« (1996, XXVI), formuliert Viktor Žmegač in der Einleitung zu seiner sehr erfolgreichen deutschen Literaturgeschichte und benennt damit zwei klassische Ziele der Literaturgeschichtsschreibung: Beschreiben und Erklären. Allerdings verschwimmen diese nicht immer so klar benannten Ziele in der Praxis häufig. Die Verwendung von Ausdrücken wie shervorbringen`, 'provozieren`, ‘führen zu<, szurückgehen auf`, >zurückführbar auf`, ১dienen` oder seine Funktion haben`, mit denen Erklä- 
rungen eher insinuiert als gegeben werden, ist sowohl in literaturwissenschaftlichen Einzeldarstellungen als auch in literaturgeschichtlichen Darstellungen im engeren Sinne Legion. Die gerade in diesem Punkt deutlich werdende verbreitete Praxis einer »Literaturgeschichtsschreibung ohne Theorie« (Buschmeier et al. 2014, 3) verweist auf ein methodologisches Forschungsdesiderat: ${ }^{1}$ Während eine Kontroverse um die Differenz zwischen Beschreibung und Interpretation sowie um die Verwendung von Beschreibungen bei der Interpretation existiert (vgl. Kindt/Müller 2003; Gittel 2015a) und die weitreichenden Auseinandersetzungen um den Unterschied von Erklären und Verstehen seit den Anstrengungen Droysens und Diltheys die Geschichte der Literaturwissenschaft als historische textinterpretierende Wissenschaft prägten, wird der Unterschied zwischen Beschreiben und Erklären mit Blick auf die Literaturgeschichtsschreibung kaum thematisiert. ${ }^{2}$

Ansätze zur Beschreibung literarischen Wandels sind keine Mangelware und können häufig auf lange Traditionen zurückblicken: Gattungsgeschichte, Problemgeschichte, Stilgeschichte, Sozialgeschichte der Literatur sowie die verschiedenen Ansätze im russischen Formalismus und Prager Strukturalismus. Dennoch ist unklar, ob die Literaturgeschichte, in der es wenigstens nach dem jetzigen Stand der Forschung keine strengen Gesetzmäßigkeiten gibt, überhaupt Erklärungen für literaturgeschichtliche Entwicklungen (im Sinne einer Antwort auf die Frage »Warum trat dieses Phänomen auf? «³) geben kann und, wenn ja, wie diese aussehen und unter welchen Bedingungen sie gelten. ${ }^{4}$ Die Unsicherheit hinsichtlich des Erklärungsbegriffs in der Literaturwissenschaft ist auch darauf zurückzuführen, dass die entsprechende wissenschaftstheoretische Diskussion bisher nur unzureichend zur Kenntnis genommen wurde: Über Jahrzehnte hinweg dominierte Hempels deduktiv-nomologisches bzw. sein später entwickeltes induktiv-statistisches Erklärungsmodell die Debatte. In Folge der erbitterten Kritik, die der Alleingeltungsanspruch dieses Modells angesichts der Vielzahl von Disziplinen, in deren Erklärungspraktiken Naturgesetze oder statistische Gesetzmäßigkeiten keine oder nur eine sehr untergeordnete Rolle spielen, heraufbeschwor, liegen seine Schwächen heute offen zutage (vgl. für einen Überblick Salmon 1989, 11-60; Woodward 2003, 152-186). Nicht vollends verabschiedet scheint jedoch der schon Hempel leitende einheitswissenschaftliche Gedanke, der in neueren philosophischen Erklärungstheorien wie dem `Unificationist Account`(Michael

$1 \mathrm{Zu}$ den Gründen für diesen Befund siehe auch die von Matthias Buschmeier 2011 angestoßene Forschungsdiskussion im Internationalen Archiv für Sozialgeschichte der deutschen Literatur.

2 Vgl. etwa die jüngsten Systematisierungsanstrengungen in Borkowski/Heine 2013.

3 Zur Ambiguität des Erklärungsbegriffs vgl. Salmon 1998a, 5f.

4 Schon 1982 spricht Jan-Dirk Müller in diesem Zusammenhang von einer offenen Frage der Literaturgeschichtsschreibung (vgl. 207). 
Friedmann, Philip Kitcher) und dem >Causal Mechanical Model`(Wesley Salmon) fortlebt. Gleichzeitig gibt es jedoch verschiedene Tendenzen zugunsten einer Pluralität von Erklärungsmodellen (vgl. Vanderbeeken 2004): die stärkere Berücksichtigung der normativen Komponenten bei der Explikation des Erklärungsbegriffs sowie der Beziehung zwischen explanatorischen Zielen einerseits und anderen Wissenschaftszielen andererseits (vgl. Koertge 1992), das stärkere Einräumen disziplin- bzw. kontextspezifischer Anforderungen an Erklärungen und Gesetzmäßigkeiten, die im jeweiligen Bereich wenigstens grundsätzlich erreichbar sein sollen (vgl. Kincaid 2004; Woodward 2005, 162ff. und 179ff.; Elster 2007, insbes. 32-51; Mitchell 2009), und die Hypothese mehrerer Arten von Kausalrelationen (vgl. Cartwright 2004, 241-243).

Insbesondere die im Rahmen dieser Pluralisierung vorgenommene Unterscheidung verschiedener Erklärungstypen wurde in der Literaturwissenschaft bisher nur unzureichend berücksichtigt. Der vorliegende Beitrag will hier Abhilfe schaffen, indem er, in einer Engführung von Theorie und Praxis, an einem konkreten Beispiel verschiedene (in der Forschung vertretene) Erklärungstypen entfaltet, die Probleme ihrer literaturwissenschaftlichen Anwendung herausstellt und ihre Reichweite auslotet. Bevor dies geschieht, sollen jedoch der Begriff >literarischer Wandel näher erläutert (Abschnitt 2), allgemeine Anforderungen an literaturgeschichtliche Erklärungen entwickelt (Abschnitt 3) und das gewählte Beispiel, die Konjunktur einer bestimmten Art des Essayismus im ersten Drittel des 20. Jahrhunderts, eingeführt werden (Abschnitt 4). Die weitere Gliederung des Aufsatzes orientiert sich an Erklärungstypen, die in der wissenschaftstheoretischen Forschung unterschieden werden: kausale und statistische Erklärungen (Abschnitt 5), intentionale Erklärungen (Abschnitt 6), funktionale Erklärungen (Abschnitt 7), teleologische Erklärungen (Abschnitt 8) und strukturale Erklärungen (Abschnitt 9).

\section{Was ist literarischer Wandel?}

Der Begriff literarischer Wandel kann unterschiedlich verstanden werden. Wenn hier wie im Folgenden von literarischem Wandel die Rede ist, so bezieht sich das im Einklang mit vielen existierenden Ansätzen auf die »Eigenschaften >der Literatur` eines Zeitabschnitts oder Eigenschaften eines Teilsektors dieser Literatur « (Krämer 2011, 86), d. h. nicht in erster Linie auf andere Aspekte des Literatursystems, das Verlagswesen, die Literaturkritik oder die Lesegewohnheiten etc., obwohl diese in den angestrebten Erklärungen eine Rolle spielen können. Zweitens bezieht sich >literarischer Wandelı nicht auf einzelne literarische Werke, sondern auf bestimmten Textsorten entsprechende Textgruppen bzw. Textrei- 
hen. ${ }^{5}$ Erst Erklärungen, die Textgruppen betreffen, scheinen einen ausreichenden Abstraktionsgrad zu haben, um wirkliche Literaturgeschichtsschreibung zu ermöglichen. Denn eine literaturgeschichtliche Darstellung, die erklären würde, vor welchem Hintergrund und mit welchen Absichten jedes einzelne (kanonisierte) literarische Werk geschaffen wurde, wäre immer noch unbefriedigend (ähnlich argumentiert Fulda 2014, 103).

Textsortenbegriffe sind systematische Ordnungsbegriffe, die es ermöglichen, »allein anhand der Merkmalskonfiguration [eines] Werks durch textnahe Prüfung auf die definierten notwendigen und alternativen Merkmale« zu ermitteln (Fricke 2010, 20), ob das Werk zu einer bestimmten Textsorte gehört oder nicht. Den bisherigen Sprachgebrauch berücksichtigende Textsortenbegriffe, die im Rahmen einer Begriffsexplikation entstehen, beruhen häufig auf so genannten »flexiblen Definitionen« (vgl. Fricke 1981, 138-150), die notwendige Merkmale mit adjunktiv verknüpften, alternativen Merkmalen kombinieren. Theoretisch ist jedoch die Stipulation unbegrenzt vieler Textsortenbegriffe, auch mit sstarren typen, denkbar, von denen aber nur ein geringer Teil durch literaturwissenschaftliche Erkenntnisinteressen $\mathrm{zu}$ motivieren ist. Textsorten unterscheiden sich von Genres als »historisch begrenzten literarischen Institution[en]« (ebd., 132), die wesentlich bestimmte dem Publikum geläufige Genresignale und an diese gekoppelte Erwartungen zur Bedingung haben.

Der Rückgriff auf den Textsortenbegriff bei der Fassung literarischen Wandels soll größtmögliche Variabilität ermöglichen: Textsortenbegriffe können sich auf unterschiedlichen Abstraktionsstufen bewegen (z. B. Roman, essayistischer Roman, essayistischer Zeitroman) und bieten gegenüber Begriffen zur Untersuchung inhaltlichen literarischen Wandels wie `Idee` und >Motiv` den Vorteil, auch formale Aspekte berücksichtigen zu können. Gegenüber dem oben erläuterten Genrebegriff oder einem ähnlichen Begriff sliterarisch-sozialer Institutionen` (vgl. Voßkamp 1977) ist der Textsortenbegriff variabler, weil er es erlaubt, literarischen Wandel sowohl mit systematisch motivierten Ex-post-Klassifikationen als auch mit historisch motivierten Klassifikationen zu untersuchen, denn »[j]eder Text eines Genres gehört derselben [...] Textsorte an« (Fricke 2010, 20). Dies schließt keineswegs aus, dass die für ein historisch-literarisches Genre wesentlichen Genreerwartungen sowie gegebenenfalls an sie gekoppelte auto-

5 Es gibt ausgedehnte Debatten um die Frage, was ein literarisches Werk ist und inwiefern es sich vom einzelnen Textexemplar unterscheidet. Da ich glaube, dass diese Debatten für meine Fragestellung sekundär sind, und der Begriff `Werkgruppe` gegenüber dem Begriff `Textgruppe irritierende Konnotationen hat, verwende ich die Begriffe `Text und `Werk « weitgehend synonym. 
renseitige Erwartungserwartungen oder rezipientenseitige Lektürepraktiken wesentlich für bestimmte Erklärungen literarischen Wandels sein können.

Literarischer Wandel sei nun die sich zeitlich ändernde >Instantiierung ‘ ${ }^{6}$ von literarischen Textsorten in dem Sinne, dass ein- und dieselbe Textsortendefinition für Zeitabschnitt $t_{1}$ eine andere Gruppe in ihm entstandener Texte herausgreift als für den Zeitabschnitt $t_{2}$. (Im Zeitabschnitt $t_{1}$ entstanden beispielsweise nur die Werke $W_{1}$ und $W_{2}$ einer bestimmten Textsorte, im Zeitabschnitt $t_{2}$ hingegen die Werke $W_{3}, W_{4}, \ldots W_{25}$.) In weniger technischer Formulierung meint literarischer Wandel also zunächst nicht mehr als die Andersartigkeit zweier Gruppen von Texten, die alle derselben Textsorte angehören, jedoch in zwei unterschiedlichen Zeitabschnitten derselben Kultur oder Gemeinschaft entstanden. Literarischer Wandel in diesem Sinne findet, solange überhaupt literarische Texte entstehen, immer statt. Besonders erklärungswürdig und interessant sind jedoch vergleichsweise abrupte oder massive Veränderungen, die durch quantitative oder qualitative Analysen näher zu beschreiben sind:

Aus quantitativen Analysen (die Textgruppe für $t_{1}$ ist kleiner oder größer als die für $t_{2}$ ) resultieren Aussagen über >Auftauchen`, >Konjunktur`, `Fortbestehen` oder >Verschwinden ' der Textsorte, in denen gerne auf Organismus-Metaphern zurückgegriffen wird (vgl. Fowler 1971). Qualitative Analysen vergleichen die beiden Textgruppen und bestimmen Unterschiede in Bezug auf Eigenschaften, die nicht ausschlaggebend für die Zugehörigkeit zur jeweiligen Textsorte sind. Sie führen zu sehr unterschiedlichen Aussagen über Tendenzen der Entwicklung der jeweiligen Textsorte, die sich idealtypisch in solche $\mathrm{zu}$ formalem Wandel (etwa die Verwendung des Alexandriners im Sonett) und inhaltlichem Wandel (etwa die Entstehung des `negativen Bildungsromans $`$ ) unterteilen lassen.

Literarischer Wandel besteht also entweder in der Veränderung der Häufigkeit des Auftretens einer Textsorte (quantitative Analyse) oder in der Veränderung der Beschaffenheit der den Textsorten jeweils entsprechenden Textgruppen (qualitative Analyse). ${ }^{7}$ Generell können Erklärungen literarischen Wandels nur so gut sein wie die ihnen zugrunde liegenden Analysen des Wandels.

\footnotetext{
6 Dieser Neologismus stammt aus dem Sprachgebrauch der Analytischen Philosophie, wo die Rede davon, dass bestimmte (komplexe) Eigenschaften von Gegenständen instanziiert (engl. instantiated) sind, meint, dass diese Eigenschaften den Gegenständen zukommen.

7 Es bleibt darauf hinzuweisen, dass der entwickelte Begriff literarischen Wandels einen Aspekt nicht erfasst, der im weiteren Sinne zum Wandel der »Eigenschaften >der Literatur eines Zeitabschnitts« (Krämer 2011, 86) gezählt werden kann: das quantitative Ausmaß, mit dem verschiedene Texte in einem Zeitraum in Umlauf gebracht werden (Auflagenstärke, Neuauflagen, Übersetzungen etc.). Eine entsprechend modifizierte, alternative Fassung >literarischen Wandels
} 


\section{Explanatorischer Pluralismus, Anforderungen an literaturgeschichtliche Erklärungen und Erklärungstypen}

Die Konsequenz aus den eingangs skizzierten Entwicklungen im Bereich der Wissenschaftstheorie ist die Entstehung eines explanatorischen Pluralismus. Aus dem Verlust des Alleinstellungsanspruchs von Erklärungsmodellen, die wesentlich an Erklärungen in der Physik orientiert sind, und aus Untersuchungen zur Pragmatik von Erklärungen resultiert die Auffassung, dass das, was als eine gute oder erfolgreiche Erklärung gilt, (zu einem gewissen Grade) wissenschaftsspezifisch ist und von (sub-)disziplinären Standards und Zielen abhängt (vgl. Godfrey-Smith 2009, 197). ${ }^{8}$ Geht man also davon aus, dass ein für die Literaturgeschichte tauglicher Erklärungsbegriff weder einfach aus einer anderen Wissenschaft übernommen, noch aus der Analyse unserer heterogenen alltagssprachlichen Verwendungsweise allein gewonnen werden kann, liegt es nahe zu fragen, welchen Anforderungen bzw. Adäquatheitsbedingungen dieser Erklärungsbegriff genügen muss. Eine solche meines Wissens bisher noch nicht unternommene Exploration der normativen Dimensionen eines literaturgeschichtlichen Erklärungsbegriffs kann heuristisch durch die Suche nach einer Ober- und einer Untergrenze angeleitet werden: $\mathrm{Zu}$ fragen ist, ab wann ein Erklärungsbegriff für die Literaturgeschichte $\mathrm{zu}$ anspruchsvoll und ab wann er $\mathrm{zu}$ anspruchslos wird, um noch von Interesse zu sein.

Weniger Schwierigkeiten bereitet dabei die Festlegung der Obergrenze. Die Position des explanatorischen Pluralismus ist wesentlich dadurch motiviert, dass bestimmte Erklärungen, insbesondere solche, die auf Gesetzmäßigkeiten im Sinne von gesetzesähnlichen Generalisierungen zielen, in bestimmten Bereichen der Sozialwissenschaften, so auch der Literaturgeschichtsschreibung, kaum oder gar nicht erreichbar zu sein scheinen. Es liegt daher nahe, die Anforderung zu formulieren, dass Erklärungen gemäß dem gesuchten Erklärungsbegriff für die Literaturgeschichte wenigstens prinzipiell für die Mehrzahl der Wandlungsphänomene, mit denen man es in diesem Bereich zu tun hat, möglich sein müssen.

ließe sich dadurch gewinnen, dass man anstelle von in einem Zeitraum entstandenen Texten, produzierte Textexemplare unter Textsorten fallen lässt.

8 Teilweise wird mit explanatory pluralism in der Forschung auch die Auffassung verbunden, dass es innerhalb einer Wissenschaft verschiedene Erklärungstypen gibt, die unter Umständen bei der Erklärung eines bestimmten Phänomens konkurrieren können (vgl. etwa Brigandt 2013). 
Wesentlich schwieriger ist die Formulierung der Untergrenze. Obwohl linguistisch orientierte Untersuchungen zur Pragmatik von Erklärungen viele vermeintliche Merkmale von Erklärungen gemäß unserem alltagssprachlichen Begriffsverständnis infrage gestellt haben, etwa die Gerichtetheit von Erklärungen, ${ }^{9}$ lässt sich ein erster Hinweis aus der traditionsreichen, alltagssprachlich verankerten Dichotomie von >Beschreibung` und >Erklärung` gewinnen. Schon Aristoteles unterscheidet und hierarchisiert bekanntlich ein bloßes Wissen des "Dass« ( $\tau$ ò ö $\tau$ ) und ein Wissen des

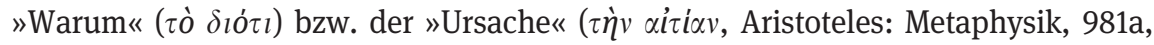
24-30) und gemeinhin sehen wir Erklärung als etwas an, das als Antwort auf eine Warum-Frage in einem unbestimmten Sinne smehr ist als Beschreibung (»Das mag ja alles so sein, aber das erklärt nicht, warum ...«). In einem ersten Zugriff kann man literaturhistorische Erklärung also bestimmen als >Beschreibung, d. h. Textgruppenbildung (inkl. quantitativer bzw. qualitativer Analyse) plus $x<$. Was aber ist dieses $x$ ?

Bezieht man diese Frage auf den Gegenstand des literarischen Wandels, ist eine erste Antwort einfacher als gedacht: Nachdem eine literarische Gruppen- bzw. Typenbildung erfolgt ist und wie im vorhergehenden Abschnitt die Häufigkeit des Auftretens der Textsorte bzw. die Veränderung ihrer historischen Ausprägungen beschrieben worden ist, muss man die Textgruppe zu einer anderen Entität in Beziehung setzen. Mit anderen Worten: Man muss die Textgruppe, die im Fokus der Erklärung stehen soll, mit einem Kontext verknüpfen. ${ }^{10}$ Es ergibt sich die $>$ Formel Erklärung = Textgruppenbildung (inkl. quantitative bzw. qualitative Analyse) + Kontextverknüpfung. Nun sieht man schnell, dass diese Gleichung nicht aufgeht, denn es gibt bekanntlich eine andere literaturwissenschaftliche Operation, die ebenfalls Texte mit Kontexten verknüpft: die Interpretation. Und obwohl es in der Gegenwart verschiedene Bestrebungen gibt, die traditionsreiche Dichotomie Verstehen bzw. Interpretation vs. Erklären zu unterlaufen, indem Interpretation als ein spezieller Typ der Erklärung begriffen wird (vgl. die Darstellung in Detel 2010),

9 Intuitionen über den Erklärungsbegriff, die auf unserem impliziten Begriffsverständnis beruhen, spielen etwa beim so genannten Symmetrieproblem, das gegen Hempels deduktiv-nomologisches Modell vorgebracht wurde, eine wichtige Rolle. Sprachpragmatische Untersuchungen beanspruchen jedoch zu zeigen, dass solche Urteile keineswegs allgemeingültig sind, sondern von der Form und dem Kontext von Warum-Fragen abhängen (vgl. van Fraassen 1980; Achinstein 1983).

10 Der Begriff `Kontext` wird hier in einem weiten Sinne verwendet, sodass er sowohl realweltliche als auch zeichenhaft verfasste Bezugsgrößen meinen kann. Die Verknüpfung von Texten mit realweltlichen, insbesondere sozialgeschichtlichen Entwicklungen oder Tatsachen wird in der Forschung auch als >Verknüpfungsproblem` bezeichnet (vgl. Schönert 2002, 337). Für einen Überblick zu literaturtheoretischen Text-Kontext-Modellen vgl. Borkowski 2015, der das TextKontext-Problem jedoch von vorneherein »als eines der Interpretation« (18), nicht der Erklärung auffasst, und vom Verknüpfungsproblem abgrenzen möchte (vgl. ebd., 9-24, 38 und 155-162). 
kann man keinesfalls davon ausgehen, dass jede Interpretation eine Erklärung sei. Vielmehr ist es mittlerweile Konsens, dass sich unter dem Begriff >Interpretation eine Vielzahl unterschiedlicher Erkenntnisinteressen und literaturwissenschaftlicher Verfahren verbergen (vgl. Hermerén 1983; Bühler 2003; Carlshamre/Pettersson 2003). Alle Interpretationen verknüpfen Texte mit Kontexten, nicht jede Verknüpfung mit einem Kontext ist jedoch eine Erklärung. Vielmehr kommt es darauf an, auf welche Weise die Texte mit dem Kontext verknüpft werden. Man kann also sagen, dass das Grundschema literaturgeschichtlicher Erklärungen lautet: Erklärung = Textgruppenbildung (inkl. quantitativer bzw. qualitativer Analyse) + Kontextverknüpfung auf die Weise $y$.

Ich vermute, dass es äußerst schwierig ist, dieses $y$, also die Art und Weise, auf die die Kontextverknüpfung erfolgen muss, in allgemeiner Form zu bestimmen, da wie ich im Folgenden zeigen möchte, in der Literaturwissenschaft unterschiedliche Erklärungstypen existieren, und es fragwürdig ist, ob sich diese unter einen einheitlichen Erklärungsbegriff subsumieren lassen. ${ }^{11}$ Demzufolge können literaturgeschichtliche Erklärungen auf verschiedene Weisen - kausal, intentional, funktional, teleologisch oder struktural - erfolgen und $y$ kann mehrere Werte annehmen. Spezifischere Anforderungen ergeben sich erst aus der Analyse dieser Erklärungstypen, an deren Analyse sowohl innerhalb der allgemeinen Wissenschaftstheorie bzw. -philosophie als auch in den fachspezifischen Wissenschaftsphilosophien bis heute gearbeitet wird. Gegenstand dieser Analysen, die sich oft an paradigmatischen Fällen von (in einer bestimmten Wissenschaft) akzeptierten Erklärungen orientieren, sind ihre Struktur, ihnen zugrunde liegende kausale Mikromechanismen sowie deren Relevanz für die Gültigkeit einer Erklärung des entsprechenden Typs. Das Verhältnis von Wissenschaftsphilosophie einerseits und Wissenschaftspraxis einer Einzeldisziplin andererseits ist dabei, wenn es produktiv ist, eines der wechselseitigen Erhellung (vgl. W. Salmon/M. Salmon 1998, 345f.).

Zwei Punkte lassen meine bis hierher sehr allgemeinen Überlegungen bewusst offen: Erstens die Frage, ob die Kontexte literarischer oder außerliterarischer Art sind und zweitens, ob die Kontextverknüpfung die Berücksichtigung der Semantik der Textgruppe erfordert. Der erste Punkt leuchtet unmittelbar ein; prima facie kann man literarischen Wandel sowohl durch innerliterarische Entwicklungen oder Gegebenheiten, etwa normbildende Werke, Leitgattungen, Gattungsdiversifizierung erklären als auch durch außerliterarische, etwa aus dem so genannten »Kontext

11 Tatsächlich hat es sich schon als Herausforderung herausgestellt, einen einheitlichen Erklärungsbegriff für die Naturwissenschaften, und sei es nur für kausale Erklärungen, zu entwickeln. Vgl. den konzisen Überblick von Cartwright, der in einem Plädoyer für »causal diversity« mündet (2004, 242). 
〉Gesellschaft`« (vgl. Mellmann 2014, 87) ${ }^{12}$ oder dem Kontext >Wissenschaft`. Hier ist eine Zwischenbemerkung angebracht: Wie anhand meines Beispiels deutlich werden wird, ist das im Hinblick auf die Interpretation notorische Beliebigkeitsproblem (woher weiß ich, welchen Kontext ich zur Interpretation eines Textes oder einer Textgruppe heranziehen soll?) im Hinblick auf die Erklärung weniger einschlägig. Denn erstens legen bestimmte Erklärungstypen bestimmte Kontexte nahe, etwa die intentionale Erklärung den Kontext `Autor`, zweitens erlauben nur wenige potentielle Erklärungskontexte gute bzw. plausible Erklärungen. Während jeder Interpretationskontext (ohne vorherige Festlegung einer Bedeutungskonzeption) prima facie brauchbare Interpretationen generiert, erlauben es nur ganz bestimmte potentielle Erklärungskontexte, ein bestimmtes Wandelphänomen zu erklären.

Der zweite Punkt, der die Frage betrifft, ob Erklärungen Bedeutungszuweisungen voraussetzen bzw. beinhalten, ist diffiziler. Denn während die Verknüpfung der Semantik literarischer Texte mit gesellschaftlichen Strukturen aus kultur- und sozialgeschichtlicher sowie wissenssoziologischer Perspektive als Kardinalproblem erscheint (vgl. Ort/Meyer 1988, 95f.; Jannidis 2000, 355f.; Ort 2011, 172), existieren vor allem in der jüngeren Forschung Tendenzen, formale Merkmale literarischer Textgruppen mit dem historischen Kontext zu verknüpfen (vgl. etwa Ajouri 2007; Nünning 2008; vgl. dazu auch King/Reiling 2014, 17f.). Hier zeichnet sich der wichtige Unterschied zwischen semantisierenden, d. h. die semantischen Gehalte der Textgruppe berücksichtigenden, Erklärungen einerseits und nicht-semantisierenden Erklärungen andererseits ab. ${ }^{13}$ Meiner Meinung nach gibt es jedoch auch einen interessanten Zwischentypus, für den in Abschnitt 7 ein Beispiel gegeben wird.

\section{Das Explanandum: die Konjunktur des fiktionalen Essayismus um 1900}

Während es seit Langem bekannt ist, dass der Essay im Zeitraum von 1890 bis 1933 im deutschen Sprachraum eine Konjunktur erfährt, wurde in jüngerer Zeit auch das eng damit zusammenhängende Phänomen der Fiktionalisierung von Reflexion, d. h. die extensive ১Übertragung`von Reflexionen auf fiktive Figuren

12 Im Gegensatz dazu plädiert Striedter dafür, literarischen Wandel immer auf Außerliterarisches zu beziehen (vgl. 1989, 74 und 81).

13 Dabei kann der semantische Gehalt der Textgruppe auch schon Teil des Textsortenbegriffs sein, etwa beim bürgerlichen Trauerspiel. 
bzw. Erzählinstanzen inspirierend beschrieben (vgl. Jander 2008). Fiktionalisierung von Reflexion beschreibt einen Aspekt des generellen Trends zur »Entgrenzung von Formen« (Kiesel 2004, 153) bzw. zur Gattungshybridisierung in der klassischen Moderne und umfasst im Bereich der Prosa zwei auffällige Entwicklungen: zum einen die gehäufte Entstehung fiktionaler Essays, zum anderen die Entstehung des so genannten `essayistischen Romans`, ein Begriff, der häufig mit der >Krise des Romans` (vgl. Kiesel 2004, 315-319) verbunden und durch den Hinweis auf die paradigmatischen Vertreter der Zwischenkriegszeit erläutert wird (vgl. bereits Frisé 1980, 80).

Um die in dem Prozessausdruck >Fiktionalisierung von Reflexion` zum Ausdruck kommende gattungsgeschichtliche Dynamik einer systematischen Untersuchung zugänglich zu machen, habe ich jüngst vorgeschlagen, Essayismus nicht länger als Sammelbezeichnung für alle Arten essayähnlicher Texte oder als Label eines quasi-universellen Denk- oder Schreibmodus zu verwenden, sondern als gattungstypologischen Oberbegriff (vgl. zum Folgenden Gittel 2015b). >Essayismus $<$ bzw. ein >essayistisches literarisches Werkı meint dementsprechend (a) ein fiktionales literarisches Werk, das (b) argumentative Satzverkettungen enthält, die als Reflexionen eines heterodiegetischen Erzählers Erzählpausen im Sinne Genettes bedingen oder Äußerungen bzw. Reflexionen von Figuren darstellen und (c) ein unsicheres Wissen prozessieren. Letzteres meint, dass für die durch die Satzverkettungen ausgedrückten propositionalen Gehalte gilt, dass ihnen entsprechende Wissensansprüche in der epistemischen Situation der Entstehungszeit des Werks kontrovers oder problematisch waren bzw. es gewesen wären.

Da es hier nicht um die Vor- und Nachteile dieses Vorschlags der Begriffsverwendung, sondern um die Erklärung der gattungsgeschichtlichen Dynamik geht, und um keine Missverständnisse aufkommen zu lassen, werde ich im Folgenden von >fiktionalem Essayismus ten sprechen, wenn ich Texte meine, die die eben genannten Bedingungen erfüllen. Das Explanandum der gesuchten Erklärung ist also die gehäufte Entstehung von fiktional-essayistischen Texten im Zeitraum von 1890 bis 1933.

Wie bereits in der Einleitung angedeutet, haben die nun folgenden Überlegungen eine mehrfache Funktion. Indem sie in der Forschung (häufig in nuce) lancierte Erklärungen der Konjunktur des fiktionalen Essayismus entfalten, tragen sie nicht nur zur Essayismus-Forschung und zur theoretischen Durchdringung der literaturgeschichtlichen Praxis bei, sondern analysieren vergleichsweise selten diskutierte, allgemeine Probleme unterschiedlicher Erklärungstypen in der Literaturgeschichtsschreibung. 


\section{Kausale Faktoren und statistische Erklärungen}

Die bekanntesten Versuche, so etwas wie Kausalerklärungen in der Literaturgeschichtsschreibung $\mathrm{zu}$ etablieren, stammen aus dem Umfeld von Wilhelm Scherer und seinen Schülern einerseits und aus dem der marxistischen Literaturtheorie andererseits (vgl. Klausnitzer 2007, 46f.; Müller 2007, 154-158). Aktualisierungsversuche solcher Ansätze in jüngerer Zeit gibt es meines Wissens nicht; vielmehr scheint es weitgehend Konsens, dass es in der Literaturgeschichte keine kausalen Erklärungen gibt. ${ }^{14}$ Interessanterweise scheintes jedoch etwas wenigstens auf den ersten Blick einer Kausalbeziehung sehr Ähnliches in der Literaturgeschichte zu geben: die Einflussbeziehung. Wenn sich die Fiktionalisierungstendenzen des Essays um 1900 teilweise auf die Rezeption Walter Paters zurückführen lassen (vgl. Burdorf 1999, 32f.; Jander 2008, 99-101), so scheinen bestimmte Prätexte bzw., im Fall der sogenannten »»Monogenese« (Gymnich 2010, 136), scheint ein Protoptyp, die Rolle von Ursachen einzunehmen, die wie Billardkugeln die Konjunktur dieser Textsorte im deutschen Sprachraum anstoßen. Um die Idee zu veranschaulichen: Pater verfasst neben ıklassischen Essays auch fiktional-essayistische Texte wie den Roman Marius the Epicurean. His Sensations and Ideas (1885) oder seine essayistischen Biographien fiktiver Personen, die von ihm selbst so genannten Imaginary Portraits (1885-1887); durch Hofmannsthal (seit 1894 Pater-Leser und nach der Jahrhundertwende selbst Autor fiktional-essayistischer Texte) auf Pater aufmerksam geworden, fährt Rudolf Kassner wenige Jahre nach Paters Tod (1894) nach Oxford, um mit dessen Lehrern und Schülern zu sprechen (vgl. Iser 2004, 143); 1900 erscheint seine Essaysammlung Die Mystik, die Künstler und das Leben, die den fiktional-essayistischen Text Stil. Ein Dialog enthält; dieser ist ein Prätext von Georg Lukács' Reichtum, Chaos und Form. Ein Zwiegespräch über Laurence Sterne (1911) und von Rudolf Borchardts Gespräch über die Formen (1905).

Auf den rational ausweisbaren Kern des >Billard-Eindrucks` weisen theoretische Analysen des Einflusskonzeptes hin, die herausstellen, dass Einfluss das Bestehen einer Kausalrelation impliziert: Wenn $a$ von $b$ beeinflusst wurde, muss es einen »direkten oder indirekten >genetischen Kontakt«" zwischen $a$ und $b$ gegeben haben (vgl. Danneberg 2008, 12). Wenn also wenigstens in Bezug auf Einflussbeziehungen von Kausalbeziehungen in der Literaturwissenschaft begründet die Rede sein kann, liegt es nahe, nach Erklärungsmöglichkeiten literarischen Wandels zu suchen, die

14 So ist Jan-Dirk Müllers Aussage aus dem Jahre 1982, dass die »Reichweite strikter Kausalerklärungen« in der Literaturgeschichte »noch enger gezogen« sei als in der Geschichtswissenschaft (198), soweit ich sehe, bis heute unwidersprochen geblieben. 
auf Einflussbeziehungen beruhen. Eine solche Möglichkeit besteht in statistischen (Teil-)Erklärungen. Dass diese Möglichkeit bisher kaum diskutiert wurde, hängt mit der Orientierung an Hempels Erklärungsmodellen zusammen, die allgemeine bzw. probabilistische Gesetzmäßigkeiten voraussetzen. Wie eingangs erwähnt, haben sowohl Hempels deduktiv-nomologisches als auch sein induktiv-statistisches Erklärungsmodell jedoch in den letzten Jahrzehnten vielfältige Kritik erfahren und zu konkurrierenden Erklärungsmodellen geführt. Diese Kritik weist, wie mir scheint, auch Wege für Erklärungen in der Literaturwissenschaft.

Einer der Kritikpunkte (neben dem Symmetrie- und Irrelevanzproblem) besteht darin, dass nach Hempels Modellen nur Phänomene erklärt werden können, die unter bestimmten Ausgangsbedingungen entweder notwendig auftreten oder eine hohe Wahrscheinlichkeit haben. Alle Erklärungen, die mit moderaten oder gar geringen Wahrscheinlichkeiten arbeiten, werden von seinem Modell daher nicht erfasst. So gibt es etwa eine Form der Lähmung (Parese), die mit einer Wahrscheinlichkeit von unter $50 \%$ bei Patienten mit unbehandelter latenter Syphilis auftritt. Tritt sie bei einem solchen Patienten auf, erklärt seine Syphilis das Auftreten, obwohl man ihr Auftreten nicht vorhersagen kann (vgl. Salmon 1998c, 56).

Ein Modell, das unter anderem zur Abdeckung solcher Fälle entwickelt wurde und auf Hempels Annahme verzichtet, dass Erklärungen (deduktive oder induktive) Schlüsse sind, ist Wesley Salmons Statistical-Relevance-Theory. Ihr liegt die Idee zugrunde, dass Erklärungen aus einer Auflistung statistisch relevanter Faktoren bestehen. Ein statistisch relevanter Faktor für das Auftreten eines Phänomens $P$ ist ein Faktor, der das Auftreten von $P$ wahrscheinlicher (nicht notwendigerweise wahrscheinlich) macht, etwa das Vorliegen unbehandelter latenter Syphilis das Auftreten von Parese.

Entsprechende statistische Erklärungen sind immer dann attraktiv, wenn die genauen Kausalketten aufgrund begrenzten Wissens oder zu geringer Daten im Dunkeln liegen. Genau das scheint mir auch der Fall bei Einflusserklärungen literarischen Wandels zu sein. Wir wissen (bislang) nicht, was im Kopf eines Autors passiert, der Walter Pater liest und irgendwann, unter Umständen Jahre später, intentional oder unbewusst, strukturell ähnliche Texte wie dieser produziert. Nichtsdestotrotz ist es eine interessante, grundsätzlich wissenschaftlich überprüfbare Hypothese, dass die Konjunktur des fiktionalen Essayismus zum Teil auf den Einfluss Paters zurückgeht. So ließen sich zwei Gruppen von Autoren - Pater-Leser und nicht Pater-Leser - vergleichend daraufhin untersuchen, welche Gruppe häufiger fiktional-essayistische Texte produziert hat. Stellt sich heraus, dass >Pater-Lektüre ein statistisch relevanter Faktor für die Produktion von fiktional-essayistischen Texten ist, so liegt der Schluss nahe, dass einige 
Pater-Leser von Paters fiktional-essayistischen Texten im Hinblick auf die Darstellungsform beeinflusst wurden.

Generell ist ein solcher Übergang von einer rein statistischen Korrelation zur Annahme einer Kausalbeziehung problematisch. Nicht umsonst hat Salmon sein Modell später dahingehend modifiziert, dass nicht nur statistische Relevanz eines Faktors $F$ für ein Phänomen $P$ für dessen Erklärung notwendig ist, sondern auch eine Rückführung dieser Relevanzbeziehung auf die Kausalbeziehung zwischen $F$ und $P$ (vgl. 1998 d, 137f.). Auf den ersten Blick sieht es nun so aus, als mache die Forderung der Beschreibung einer Kausalkette Salmons Modell genauso unattraktiv für die Literaturwissenschaft wie Hempels Modelle. Indes verfügt die Literaturwissenschaft mit der Einflussrelation über eine Vorstellung, die genau diese Lücke füllen kann: Die Pater-Leser produzieren deshalb fiktional-essayistische Texte, weil sie Kontakt mit Paters Werken hatten und diese Werke auf eine ganz bestimmte Weise verarbeitet haben, die ihre eigene Produktionsweise geprägt hat. Gewiss ist diese Vorstellung für eine Autorengruppe ein Blackbox-Modell, aber erstens ist diese Blackbox grundsätzlich aufklärbar, etwa indem man Kassners Faszination für Pater näher untersucht (vgl. Iser 2013, 144f.), zweitens ist es ein Modell, das einen kausalen Zusammenhang zwischen $F$ und $P$ etabliert. Dies wird deutlich, wenn man die hier nur projektierte empirische Untersuchung mit einer möglichen anderen vergleicht. Nehmen wir an, es ließe sich zeigen, dass Kaffeetrinker signifikant häufiger fiktional-essayistische Texte produzieren als Teetrinker. In diesem Fall gäbe es offenbar nicht eine einzige sinnvolle Hypothese über den Kausalzusammenhang. Nicht jeder statistisch relevante Faktor ist also auch ein kausal relevanter Faktor. Statistische Relevanzbeziehungen allein sind nicht ausreichend, um einen Kausalzusammenhang anzunehmen, aber sie sind ein Hinweis darauf, wo er zu suchen ist (vgl. W. Salmon/M. Salmon 1998, 343; Salmon 1998b, 350).

Selbstverständlich sind die vorstehenden Überlegungen viel eher eine Beschreibung eines künftigen Arbeitsfeldes für einen mit allen Wassern der Stochastik gewaschenen Literaturhistoriker, der sich der Werkzeuge der Computerphilologie bedient, als eine Beschreibung bestehender Erklärungspraxis. Ein solcher `Zukunftsphilologe`sollte auch in der Lage sein, mögliche Fehlerquellen auszuschließen, etwa die Möglichkeit, dass der vermeintlich kausale Faktor und das $\mathrm{zu}$ erklärende Phänomen eine gemeinsame Ursache haben, im skizzierten Beispiel etwa die Lektüre Platons (die zur Pater-Lektüre führen kann, da Pater 1893 gesammelte Vorlesungen unter dem Titel Plato and Platonism publiziert); ${ }^{15}$

15 Beruhen sowohl >fiktionaler Essayismus` als auch >Pater-Lektüre` auf dem Einfluss durch Platon-Lektüre, so wird durch Berücksichtigung dieses Faktors der Faktor Pater-Lektüre statistisch irrelevant (screening-off-Effekt), d. h. zwei Vergleichsgruppen, in denen alle Platon gelesen 
oder die Möglichkeit, dass Pater-Lektüre (als Teil einer sogenannten INUS-Bedingung (J.L. Mackie)) nur in Kombination mit einem oder mehreren anderen Faktoren (etwa Interesse an neuen Ausdrucksmöglichkeiten) zur Produktion fiktional-essayistischer Texte führt.

Festzuhalten ist jedoch: Im Zusammenspiel von biographischen und einflussgeschichtlichen Untersuchungen sowie statistischen Analysen ist es prinzipiell möglich, kausale Faktoren literarischen Wandels zu bestimmen und entsprechende statistische (Teil-)Erklärungen zu geben, obwohl die allermeisten dieser Faktoren nicht durch Gesetzmäßigkeiten oder gesetzesähnliche Generalisierungen mit den Phänomenen verknüpft sein dürften.

\section{Intentionale Erklärungsansätze}

Wenn es im letzten Abschnitt wesentlich darum ging zu zeigen, dass die Literaturwissenschaft durch ein verzerrtes Bild von Erklärungen in den Naturwissenschaften ihre eigenen Möglichkeiten unterschätzt hat, so konzentriert sich dieser Abschnitt auf einen Erklärungstyp, der den meisten Literaturwissenschaftlern, auch aus ihrer Praxis, vertraut sein dürfte: intentionale Erklärungen. Diese sind wissenschaftstheoretisch vergleichsweise gut erforscht. Sie stellen einen Subtyp der mentalen Erklärungen dar, die auf geistige Zustände des Handelnden wie Meinungen, Gefühle oder Absichten rekurrieren, und haben im Allgemeinen folgende Struktur: Ein Subjekt $S$ tut $x$, weil (1) $S$ das Ziel $Z$ hat und (2) $S$ glaubt, dass $x$ unter den gegebenen Umständen ein adäquates Mittel bzw. ein geeigneter Beitrag zum Erreichen von $Z$ ist (vgl. Beckermann 1977, 45-57).

Da literarischer Wandel sich auf Textgruppen bezieht, intentionale Erklärungen jedoch auf das Handeln einer Person (hier die Textproduktionshandlung eines Autors), lässt sich mit ihrer Hilfe nur die Entstehung von Einzeltexten einer Textgruppe erklären. Die Erklärung literarischen Wandels mithilfe von intentionalen Erklärungen setzt daher methodisch ihre Akkumulation voraus. Möglicherweise lassen sich jedoch nach der Akkumulation im Pool der Einzelerklärungen bestimmte Muster feststellen, die eine strukturell einfachere Erklärung zulassen als die Verknüpfung aller Einzelerklärungen durch logische Konjunktionen. In jedem Fall ist das Verfahren sehr aufwändig und in der Praxis wohl nur von Forschergruppen durchführbar. Aus methodologischer Sicht hat ein solches Vorgehen jedoch auch Vorteile. Erstens entspricht es der

haben, jedoch nur in einer Pater, dürften keinen relevanten Unterschied hinsichtlich der Häufigkeit fiktional-essayistischer Texte zeigen. 
Doktrin des methodologischen Individualismus, die postuliert, dass akteursbzw. ereignisbasierte Erklärungen faktenorientierten Erklärungen vorzuziehen sein (vgl. Elster 2007, 9-13). Zweitens ermöglichen intentionale Erklärungen ein vergleichsweise textnahes Vorgehen, da sie häufig auf poetologische Äußerungen und Selbstkommentare der jeweiligen Autoren rekurrieren.

Allerdings ermöglichen selbst umfangreiche poetologische Äußerungen nicht in gleichem Maße plausible intentionale Erklärungen, da ihre Plausibilität wesentlich von der Spezifizität der poetologischen Äußerungen abhängt. Dies soll an drei poetologisch sehr beschlagenen Autoren von fiktional-essayistischen Texten kurz veranschaulicht werden: ${ }^{16}$

$\mathrm{Zu}$ wenig spezifisch und daher ungeeignet, um die eigene fiktional-essayistische Schreibpraxis (Von der Armut am Geiste, 1911/1912; Reichtum, Chaos und Form, 1911) zu erklären, ist die Essaytheorie von Georg Lukács, die als »wenig ausgearbeitete Brieffiktion« (Jander 2008, 107, Anm. 350) selbst in einem fiktional-essayistischen Text (Über Form und Wesen des Essays, 1911) niedergelegt ist. Denn erstens reflektiert Lukács nicht über den Unterschied zwischen fiktionalen und nicht-fiktionalen Essays und zweitens steht seine Theorie, dass jeder Essay eine »ursprüngliche und tiefe Stellungnahme zum Ganzen des Lebens « ist (1911, 38), in einer Spannung zu den vielfältigen Relativierungseffekten, die die Fiktionalisierung von Reflexion mit sich bringt (vgl. Gittel 2016).

Robert Musils poetologische Äußerungen sind spezifischer als die von Lukács, decken jedoch nicht die ganze Breite seiner fiktional-essayistischen Praxis ab, wie sie sich im Mann ohne Eigenschaften manifestiert. Vor dem Ersten Weltkrieg schreibt er essayistischen Passagen in der Fiktion eine Funktion bei der Vermittlung einer ılebendigen Erkenntnis` zu (vgl. Gittel 2013, 153-168). Nach seiner ısoziologischen Wende` dient das Essayistische nicht länger der Darstellung von Gefühls-Gedanken-Komplexen, sondern der Figurencharakterisierung und -typisierung (vgl. Musil 2009a, Heft 8/6; 2009b, II/1/142). ${ }^{17}$ Allerdings scheinen Musils Äußerungen nach dem Ersten Weltkrieg lediglich die reflexiven Passagen, die Figuren $\mathrm{zu}$ attribuieren sind, $\mathrm{zu}$ erklären (Ideen »Personen in den Mund« legen), nicht jedoch die vielfältigen Passagen, die dem Erzähler zuzuschreiben sind.

16 >Fiktionaler Essayismus ist eine Ex-Post-Klassifikation, die den Autoren nicht zu Verfügung stand. Dennoch kann sie in entsprechenden Erklärungen verwendet werden, da sie den Autoren nicht zugeschrieben werden muss. Zum Unterschied zwischen Anwendung und Zuschreibung in diesem Zusammenhang vgl. Spoerhase 2007, 194-196.

17 Beide Begründungen für die Fiktionalisierung von Reflexion zusammenzudenken versucht Wolf 2011, 108f. Wenig überzeugende, da durch Selbstkommentare Musils kaum belegbare Hypothesen zum Essayismus als kalkuliert publikumswirksames Element finden sich in Henninger 1980, 101, und Moser 1980, 175-178; kritisch dazu Fanta 2000, 314, Anm. 5. 
$\mathrm{Zu}$ guter Letzt noch ein Beispiel einer erfolgreichen intentionalen Erklärung: Im Rahmen seiner kontrovers diskutierten Konzeption des »erkenntnistheoretischen « bzw. "polyhistorischen Romans «, ${ }^{18}$ die Hermann Broch vor dem Hintergrund einer Unzufriedenheit mit dem Positivismus einerseits und dem Postulat einer »Erkenntnispflicht « der Literatur andererseits entwickelt $(1957,161)$, finden sich auch Überlegungen, die die Integration des Essays Zerfall der Werte in den dritten Teil der Schlafwandler-Trilogie betreffen. Die Integration des Essays dient gemäß Broch - sehr verkürzt gesagt - einer Konzeption, die idealiter Totalität durch eine subtiles Neben- und Nacheinander von perspektivisch bedingten Weltzugriffen vermittelt. Der Wertzerfall-Essay, der durch seine Zuschreibung an die Figur Dr. Bertrand Müller lediglich als ein möglicher Zugriff auf Welt markiert wird, ist innerhalb der Fiktion zentraler Baustein einer »rationale[n] Annäherung an jene irrationale Gesamterkenntnis« (ebd., 68), die Broch die dichterische nennt.

Dass der intentionale Erklärungsansatz lediglich bei einem der drei Autoren fiktional-essayistischer Texte fruchtbar ist, obwohl von ihnen elaborierte poetologische Äußerungen vorliegen, macht deutlich, wie unwahrscheinlich es ist, dass sich ein überindividuelles Phänomen wie die Konjunktur einer Textsorte durch Akkumulation intentionaler Erklärungen deuten lässt. Dafür sind selbst umfangreiche poetologische Äußerungen häufig entweder zu unspezifisch (z. B. Lukács) oder zu spezifisch, d. h. sie erklären nur Teile des Phänomens (z. B. Musil).

\section{Funktionale Erklärungsansätze}

\subsection{Struktur und Probleme funktionaler Erklärungen}

Obwohl funktionsgeschichtliche Ansätze in der Literaturwissenschaft Konjunktur haben, wird die Frage nach ihrem explanatorischen Potential kaum explizit gestellt. $^{19}$ Während explanatorische Konnotationen in diversen funktionsgeschichtlichen Studien (die auf textinterne und/oder textexterne Funktionen

18 Vgl. u. a. Kreutzer 1966; Menges 1970; Vollhardt 1986.

19 So wird in einem Überblicksartikel zu funktionsgeschichtlichen Ansätzen in der Literaturwissenschaft vermutet, dass der "notorische Mangel an begrifflicher Schärfe in Diskussionen über Funktionen(en) literarischer Texte« mit der Suggestion von »klare[n] kausalen Relationen zwischen Ursache und Wirkung« zusammenhängt (Gymnich/Nünning 2005, 3), ohne jedoch die explanatorische Frage im Weiteren zu thematisieren. 
abheben $^{20}$ ) mehr oder minder reflektiert >mitschwingen ${ }^{21}$ erheben Ansätze anderer Provenienz, die sich unter dem Label Problemgeschichte subsumieren lassen, teilweise explizit Ansprüche auf funktionale Erklärungen. Was also bedeuten Funktionszuschreibungen an Textreihen, welche Struktur haben sie, wie lassen sie sich rechtfertigen und welche Probleme treten dabei im Hinblick auf literarischen Wandel auf?

Im Gegensatz zu intentionalen Erklärungen, die voraussetzen, dass die jeweiligen Autoren bestimmte Problemkonstellationen wahrnehmen, auf die sie mit poetologischen und poetischen Innovationen reagieren, sind funktionale Erklärungen besonders dann attraktiv, wenn intentionale Erklärungen scheitern (vgl. Wray 2002, 87). Funktionale Erklärungen arbeiten mit der Annahme von unbemerkten oder lediglich von Einzelakteuren wahrgenommenen Nutzeffekten, wobei sie das Vorliegen eines bestimmten Phänomens durch seinen Nutzen für den Fortbestand einer beliebigen Entität, etwa eines Organismus, einer Gruppe von Individuen oder eines Systems, erklären. Klassische Beispiele für funktionale Erklärungen finden sich in der Evolutionstheorie, die auf dem Nutzen eines bestimmten phänotypischen Merkmals für das Überleben beruhen.

In den Sozialwissenschaften haben funktionale Erklärungen, folgt man Daniel Little, generell folgende Struktur, wobei $P$ eine Praktik, $S$ ein soziales System und $B$ einen Nutzeffekt (benefit) repräsentieren soll (vgl. 1991, 94):

1. $\quad P$ persists in $S$

2. $P$ has the disposition to produce $B$ in the circumstance of $S$

3. $\quad P$ persists in $S$ because it has the disposition to produce $B^{22}$

Die Struktur funktionaler Erklärungen in dieser Weise anzugeben, ist selbst schon Ausdruck einer Parteinahme in einer wissenschaftstheoretischen Debatte. Bedingung 3 oder ähnliche Bedingungen, die bedeuten, dass die Tatsache, dass die Praktik $P$ die Disposition hat, $B$ hervorzubringen, das Fortbestehen von $P$ verursacht, werden nämlich seit geraumer Zeit mit dem Anspruch formuliert, funktionale Erklärungen in den Sozialwissenschaften von Scheinerklärungen zu unterscheiden (vgl. Elster 1994, 407; 2007, 14f.).

20 Zur Unterscheidung von internen und externen Funktionen vgl. Fricke 1981, 91-100. Eine Liste der äußerst unterschiedlichen Gegenstände, die mit dem Funktionsbegriff traktiert werden, findet sich in Sommer 2000, 321.

21 Nur wenige funktionsgeschichtliche Studien lösen den Funktionsbegriff bewusst von seinen explanatorischen Konnotationen. Eine Ausnahme bildet Seeber 2005, 80; ähnlich Fluck 2005, 31f. 22 Die drei Bedingungen entsprechen der Erläuterung des Beispiels durch Little, in der er im Gegensatz zu den Bedingungen, wo er zwischen einer »disposition to produce $A$ « (Bed. 2) und einer »disposition to produce $B$ « (Bed. 3) unterscheidet, $A$ und $B$ jedoch gleichsetzt. 
Die Anwendung von Bedingung 3 im konkreten Fall erfordert zu zeigen, wie genau, mittels welcher Feedback-Mechanismen ${ }^{23}$ die Praktik $P$ als Ursache durch die (indirekte) Wirkung $B$ ihr eigenes Fortbestehen sichert, ${ }^{24}$ und so den klassischer Einwand zu entkräften, funktionale Erklärungen würden das Verhältnis von Ursache und Wirkung verdrehen. In der Biologie besteht dieser Feedback-Mechanismus im evolutionstheoretisch verbürgten Mechanismus der natürlichen Selektion: Die Wirkung (das Angepasstsein eines Organismus) hat einen Einfluss auf die Wahrscheinlichkeit des Auftretens der Ursache (Gen), da angepasste Organismen ihre Gene mit höherer Wahrscheinlichkeit weitergeben. Die Fragwürdigkeit funktionaler Erklärungen in den Sozialwissenschaften beruht für viele Wissenschaftstheoretiker auf dem Fehlen eines (generellen) zur natürlichen Selektion analogen Mechanismus der sozialen Selektion (vgl. Wray 2002, 73-77). Auf diesen Punkt komme ich in Abschnitt 7.4 zurück.

Ein sehr allgemeiner Einwand gegen funktionale Erklärungen lautet, sie seien zur Erklärung von sozialem bzw. literarischem Wandel nicht geeignet, da sie stets nur erklärten, welche Leistung ein Element für den Fortbestand eines Systems erbringt (vgl. Eibl 1991, 351). Diesem schon von der Frankfurter Schule gegen Parsons und Luhmann erhobenen Vorwurf lässt sich dadurch begegnen, dass man Bedingung 3 folgendermaßen dynamisiert: Der benefit $B$ besteht darin, dass er ein zu $t_{O}$ bestehendes Defizit oder einen Bedarf des Systems zu $t_{1}$ behebt bzw. deckt. Wandel allgemein lässt sich also funktional erklären, indem man das zu erklärende Phänomen als das Beheben eines früheren defizitären Systemzustandes konzeptualisiert.

\subsection{Funktionale Erklärungen der Konjunktur des fiktionalen Essayismus}

Wie sehen nun mögliche Nutzeffekte der Praktik der Produktion fiktionalessayistischer Texte aus? Simon Jander hat für eine Teilmenge fiktional-essayistischer Texte die These entwickelt, dass die »zentralen Funktionen der fiktionalen Essayformen« in der »Ermöglichung einer poetischen Epistemologie und einer wirkungsästhetisch ambivalenten Didaktik« bestehen, die Verfahren

23 Zur beachtlichen Konjunktur des Begriffs des ısocial mechanism ten generell vgl. Hedström/Ylikoski 2010, 49-67.

24 In die gleiche Richtung geht der Vorschlag, funktionale Erklärungen als Spezialfall kausaler Erklärungen, als kausale Erklärungen mit einer Kausalbehauptung zweiter Ordnung zu begreifen (vgl. Steel 2005, 941-952). 
der »Leser-Einbindung " und »Leser-Distanzierung " verbindet (2008, 369; vgl. dazu ebd., 360-369). Insbesondere was die poetische Epistemologie betrifft, ließe sich diese These auch auf den essayistischen Roman des Untersuchungszeitraums ausweiten. ${ }^{25}$ Was Janders lesenswerten Ausführungen fehlt, um ein wirkliches Angebot einer funktionalen Erklärung sein zu können, ist jedoch eine Antwort auf die Frage, warum eine poetische Epistemologie im Untersuchungszeitraum nottat bzw. wo der Bedarf für sie herrührte. Da er diese Frage nicht thematisiert, bleibt unklar, inwiefern die Leistung, die die Textsorte erbringt, in irgendeiner Weise ihr Auftreten erklärt.

Alternativ scheint mir vor allem die Hypothese aussichtsreich, dass fiktionaler Essayismus der Fortführung der Auseinandersetzung mit weltanschaulichen Fragen unter den Bedingungen des weltanschaulichen Pluralismus dient. ${ }^{26}$ Fiktionaler Essayismus ist demnach eine Reaktion auf einen komplexen denkgeschichtlichen Umbruch, von einer Situation, in der Weltanschauungen als Weltbeschreibungsentwürfe wahrgenommen werden, zu einer, in der sie als perspektivenabhängige Darstellungen der Welt aufgefasst werden, die einen subjektiven und letztlich nicht begründungsrational einholbaren Weltzugang exemplifizieren, was schließlich zu einer »wahre[n] Hochflut an Typisierungsliteratur» von Weltanschauungen und geschichtsphilosophischen und/oder morphologischen Deutungen von >Kulturstufen führt (Bollnow 1967, 5). ${ }^{27}$

Formuliert man diesen Erklärungsansatz nach dem oben erläuterten Schema aus, lassen sich exemplarisch Probleme verdeutlichen, die zum Teil auf der Eigenart funktionaler Erklärungen beruhen, zum Teil aber auch auf Besonderheiten ihrer Anwendung in der Literaturwissenschaft:

1. Fiktionaler Essayismus herrscht im ersten Drittel des 20. Jahrhunderts im deutschen Sprachraum vor.

2. Fiktionaler Essayismus hat die Disposition, den Bedarf nach Auseinandersetzung mit weltanschaulichen Fragen unter den Bedingungen des zunehmend sichtbar werdenden Weltanschauungspluralismus zu decken.

25 Vgl. die Ausführungen von Dittrich 2009 zu der komplexen Auseinandersetzung mit den Defiziten »rationale[r] Erkenntnis« (253) im essayistischen Roman sowie die Hinweise in Jander 2008, 365, Anm. 974.

26 Nicht weiter verfolgt wird hier die These eines Funktionsverlusts des klassischen Essays zu Beginn des 20. Jahrhunderts (vgl. Schlaffer 1975, insbes. 144 und 169).

27 Diese neue denkgeschichtliche Situation ist keineswegs eine solche, in der es illegitim wäre, in nicht-fiktionalen Kontexten weltanschauliche Behauptungen aufzustellen. Im Gegenteil bewerten aufmerksame Beobachter die außerakademische Diskurslandschaft als äußerst unkritisch (vgl. Hesse 1934, 650; Musil 2009c, 852; Tucholsky 1922, 237). Es handelt sich also um keine einfache `Schutzraumfunktion der Fiktion. 
3. Fiktionaler Essayismus herrscht vor, weil er die Disposition hat, den Bedarf nach Auseinandersetzung mit weltanschaulichen Fragen unter den Bedingungen des zunehmend sichtbar werdenden Weltanschauungspluralismus zu decken.

Für die erste Teilthese wurde bereits in Abschnitt 4 argumentiert. Was die zweite angeht, lässt sich zeigen, dass der fiktionale Essayismus unsicheres Wissen in einem semi-assertiven Modus prozessiert, d.h. dass die unsicheren Wissensansprüche zwischen Behauptungen gegenüber dem Leser und innerliterarischer Bedeutung oszillieren (vgl. Gittel 2015b). Denn wenn fiktional-essayistische Texte das »Wirkpotential« (Sommer 2000, 328) haben, den Leser assertiv mit weltanschaulichen Behauptungen und Argumentationen zu konfrontieren, haben sie ipso facto die Disposition, den Bedarf nach weltanschaulicher Auseinandersetzung zu decken.

Anhand von Bedingung 2 und Bedingung 3 möchte ich im Folgenden zwei allgemeine Schwierigkeiten für funktionale Erklärungen im Bereich der Literaturgeschichte erläutern. Beide Schwierigkeiten sollen jeweils in Zusammenhang mit einem bestimmten Forschungsprogramm diskutiert werden. Die erste Schwierigkeit besteht in der Rede von einem >Bedarf nach Auseinandersetzung ‘ in Bedingung 2, über die versucht wird, dem fiktionalen Essayismus einen gesellschaftlichen Nutzen zuzuschreiben, obwohl Literatur zu Recht als »vom Lösungszwang entlastetes Organon der symbolisch generalisierten Reflexion« beschrieben wurde (Eibl 1995, 33). Diese Schwierigkeit soll in Auseinandersetzung mit dem Ansatz der Problemgeschichte diskutiert werden. Die Begründung von Bedingung 3 wirft die zweite Schwierigkeit auf, nämlich die Frage nach einem FeedbackMechanismus, der den gesellschaftlichen Nutzen des fiktionalen Essayismus an die gehäufte Entstehung fiktional-essayistischer Texte rückkoppelt. Diese Schwierigkeit soll in Auseinandersetzung mit Theorien sozialer Selektion erfolgen.

\subsection{Die erste Schwierigkeit funktionaler Erklärungen: Die svom Lösungszwang entlasteter Literatur und das Postulat gesellschaftlicher `Bedürfnisse،}

Texte reagieren auf realweltliche Probleme, Texte antworten auf Probleme und Texte verhandeln mögliche Lösungen solcher Probleme. Schon an solchen Redeweisen wird deutlich, dass die Problem-Lösungs-Figur eine Antwort auf die Frage verspricht, warum ein bestimmter Text bzw. bestimmte Typen von Texten zu einer bestimmten Zeit entstanden. Und tatsächlich ist die Problemgeschichte bzw. die problemgeschichtliche Ideengeschichte einer der wenigen literaturwissenschaftlichen Ansätze, der explizit den Anspruch auf (funktionale) Erklärung 
literarischen Wandels erhebt. So verweist Wilhelm Voßkamp bei der Erläuterung seiner Konzeption, der zufolge »historische Gattungsinstitutionalisierungen « in einem »funktionalen Zusammenhang mit historischen Bedürfnissen oder Bedürfnisdispositionen « stehen, in einer Fußnote auf den Typus der »funktionalistischen Erklärung « (1982, 184 und 193, Anm. 5). So propagiert Karl Eibl seinen einflussreichen problemgeschichtlichen Ansatz explizit als Antwort auf das Defizit der Systemtheorie, historischen Wandel nicht erklären zu können, und propagiert eine »historische Systemtheorie«. Diese begreift einen Systemzustand als »das vorläufige Ergebnis von Selektionsprozessen in der Zeit«, um ihn »funktional erklären zu können« $(1991,354) .{ }^{28}$ Ohne dass hier Eibls ganze Konzeption im Detail erläutert werden könnte, ist die Schnittstelle zwischen jener historischen Systemtheorie und der Problemgeschichte zu markieren: »Problemlösungen [...] können systemtheoretisch als Funktionen gedeutet werden.« (ebd., 351; vgl. auch die Ausführungen zu »strukturellen Kopplungen« in Eibl 2000) Ein anderer im deutschen Sprachraum sehr einflussreicher problemgeschichtlicher Ansatz stammt von Michael Titzmann. Im Gegensatz zu Eibls ambitioniertem Vorschlag, der Wissenschafts- und Literaturgeschichte nur als einen Ausschnitt des Wandels »exosomatische[r] Überzeugungssysteme« (Eibl 1991, 360) überhaupt behandelt, ist Titzmanns Ansatz in erster Linie zur Erklärung literarischen Wandels konzipiert. Auch er möchte den Wandel von einem Systemzustand zum anderen mittels einer »funktionale[n] Erklärung « erläutern: »Der Wandel erfüllt eine Funktion, und die angestrebte Erfüllung dieser Funktion erklärt den Wandel.« (Titzmann 1991, 430, Hervorhebung B.G.; vgl. auch 2010, insbes. 332) Aufgegriffen und methodisch-begrifflichen Klärungen zugeführt wurde der Erklärungsanspruch der Problemgeschichte in jüngerer Zeit von Dirk Werle und Katja Mellmann (vgl. Werle 2009, Mellmann 2014, insbes. 106-111 sowie die Beiträge der dadurch initiierten Forschungsdiskussion in der Scientia Poetica 2009/2010 und Werle 2014).

Konzipiert man funktionale Erklärungen in der Literaturgeschichte mithilfe der Problem-Lösungs-Figur, stellt die Tatsache, dass literarische Texte in aller Regel keine Probleme lösen (vgl. Eibl 1995, 33; 2000, 182), eine ernstzunehmende Schwierigkeit dar. Denn aus dem Befund, dass ein Text bzw. eine Textgruppe lediglich >anstrebt` oder >versucht`, ein Problem zu lösen, etwa indem er bzw. sie es thematisiert, lassen sich schwerlich funktionale Erklärungen ableiten: Wenn

28 Die sehr knappen Formulierungen Eibls lassen außen vor, dass wenigstens die Systemtheorie Luhmannscher Prägung durchaus das Ziel verfolgt, »evolutionäre Zusammenhänge« (Schützeichel 2003, 176), vornehmlich solche zwischen Gesellschaftsstruktur und Semantik, aufzuzeigen (vgl. etwa Luhmann 1990, insbes. 183f. und 209f.; 2001, 413-594). 
Archäologen bei Ausgrabungen viereckige Räder finden, wird man ihre Existenz nicht mit ihrer Funktion für die Fortbewegung erklären. Man könnte höchstens die Hypothese aufstellen, dass der Erfinder das Fortbewegungsproblem lösen wollte, dies wäre jedoch ein intentionaler Erklärungsansatz.

Will man sich nicht mit intentionalen Erklärungen für die Produktion einzelner Texte des Korpus begnügen, liegt es nahe von gesellschaftlichen »[B]edürfnissen« (vgl. Voßkamp 1977, 34, vgl. auch 32) oder einem gesellschaftlichen Bedarf der Thematisierung bzw. Reflexion dieser Probleme auszugehen. ${ }^{29}$ Die entsprechenden Texte erfüllten dann eine gesellschaftliche Funktion, indem sie bisher unterreflektierte Probleme thematisieren. Aus diesem Vorschlag ergeben sich zwei wichtige Konsequenzen für problemgeschichtliche Erklärungsansätze:

(a) Da der Ausdruck >Bedarf nach Thematisierung/Reflexion eines Problems kaum anders als intentional interpretierbar ist, fallen lediglich ex post wahrnehmbare Probleme aus dem Pool möglicher Probleme heraus.

(b) Unterscheidet man konsequent zwischen dem außertextuellen Problem, das in einem Text bzw. einer Textreihe thematisiert wird, und dem Bedarf der Thematisierung/Reflexion dieses Problems als Metaproblem, wird deutlich, dass eine Problemgeschichte mit explanatorischem Anspruch literarische Texte in aller Regel (d.h. wenn diese keine Probleme lösen) als Lösungen des Metaproblems konzipieren muss. Am Text bzw. der Textreihe sablesen kann man jedoch bestenfalls das Problem, nicht das Metaproblem, denn es ist durchaus denkbar, dass Texte Probleme reflektieren, für deren Reflexion es keinen nennenswerten gesellschaftlichen Bedarf gibt. Dies hat eine methodologische Konsequenz: Das Metaproblem sollte unabhängig von den Texten der problemgeschichtlich zu untersuchenden Textreihe nachgewiesen werden. ${ }^{30}$

Auch die zweite Bedingung der vorgeschlagenen funktionalen Erklärung des fiktionalen Essayismus arbeitet mit dem Begriff `Bedarf`, allerdings handelt es

29 Eibl etwa suggeriert einen solchen Bedarf, wenn er schreibt, seit dem letzten Drittel des 18. Jahrhunderts bearbeite die Dichtung »Problembereiche, die von den offiziellen Problemlösungsinstanzen nur unzureichend und kalmierend behandelt worden waren« (Eibl 1991, 364, Hervorhebung B.G.).

30 Diese Überlegungen werfen folgende Frage auf: Können auch in Fällen, in denen kein Metaproblem eine Rolle spielt, das im Text thematisierte Problem und das Problem, auf das der Text ıantwortetı, auseinanderfallen? Es besteht der Verdacht, dass die Attraktivität des problemgeschichtlichen Ansatzes u. a. darauf beruht, dass er eine Ineinssetzung von Interpretationskontext und Erklärungskontext nahelegt. 
sich bei dem im fraglichen Zeitraum vorhandenen Bedarf nach Auseinandersetzung mit weltanschaulichen Fragen (vgl. Sloterdijk 1995) um kein Metaproblem im eben beschriebenen Sinne. Vielmehr ist der nicht gedeckte Bedarf nach Auseinandersetzung mit weltanschaulichen Fragen selbst das Problem, welches die Konjunktur der fiktional-essayistischen Texte löst, weil ihr spezielles ästhetisches Verfahren (die Fiktionalisierung von Reflexion) weltanschauliches Wissen in einem semi-assertiven Modus prozessiert, der beim Leser eine kognitive Auseinandersetzung mit diesem Wissen provoziert.

Das Beispiel ist jedoch nicht nur aufschlussreich, weil es zeigt, dass es funktionale Erklärungen geben kann, die mit der Prämisse arbeiten, dass eine Textsorte ein Problem löst, sondern auch, weil es veranschaulicht, dass eine Erklärung literarischen Wandels durch Verknüpfung mit dem Kontext >Gesellschaft` nicht zwangsläufig über komplexe Bedeutungszuweisungen an die entsprechende Textgruppe erfolgen muss. Wesentlich für die Erklärung ist nicht die Beobachtung, dass alle Texte ein bestimmtes Problem thematisieren (tatsächlich wird Weltanschauungspluralismus nur in wenigen der fiktional-essayistischen Texte des Untersuchungszeitraums thematisiert), sondern die Analyse des ästhetischen Verfahrens der Textsorte und seines Wirkpotentials sowie die Beobachtung, dass das verarbeitete unsichere Wissen in großem Maße weltanschauliches Wissen ist. Obwohl letzteres eine Berücksichtigung der Semantik der Texte über die semantischen Merkmale der Textsorte fiktionaler Essayismus hinaus erfordert, sind für die funktionale Erklärung keine besonders komplexen oder umfassenden Bedeutungszuweisungen im Sinne von Einzeltextinterpretationen erforderlich. Es lässt sich daher von einer schwach-semantisierenden Erklärung sprechen. Im Mittelpunkt steht nicht der ideell-thematische Gehalt der Texte oder ihre Welthaltigkeit, sondern wie sich diese Texte auf Wirklichkeit beziehen und zum Wissenssystem bzw. der epistemischen Situation der Zeit verhalten.

\subsection{Die zweite Schwierigkeit funktionaler Erklärungen: Soziale Selektion als Feedback-Mechanismus?}

Offensichtlich bildet die Bedingung 3 (»Fiktionaler Essayismus herrscht vor, weil er die Disposition hat, den Bedarf nach Auseinandersetzung mit weltanschaulichen Fragen unter den Bedingungen des zunehmend sichtbar werdenden Weltanschauungspluralismus zu decken.«) die Crux der ganzen Argumentation und die am schwersten zu belegende Behauptung. Ohne sie ist die funktionale Erklärung nicht valide. Wie also lässt sie sich plausibilisieren? Vor dem Hintergrund der Tatsache, dass die erfolgreichsten funktionalen Erklärungen aus der Biologie stammen, liegt es nahe, einen zur natürlichen Selektion analogen Mechanismus der sozialen 
Selektion anzunehmen: ${ }^{31}$ Wie in der Evolution das Angepasst-Sein eines Organismus an seine Umwelt die Weitergabe seines Genmaterials wahrscheinlicher macht, soll die Funktionalität einer gesellschaftlichen Entität (Praxis/Institution/Teilsystem/System) ihr Fortbestehen bzw. ihre Reproduktion wahrscheinlicher machen. Tatsächlich wird dieser Vorschlag, der seit längerer Zeit kritisch in der Soziologie diskutiert wird (vgl. Stichweh 1999), in jüngster Zeit in verschiedenen Forschungsfeldern, etwa der Vergleichenden Soziologie (vgl. Runciman 2009), der Ökonomie ${ }^{32}$ oder Organisationstheorie (vgl. Gill 2011) mit neuem Eifer verfolgt. In der Literaturwissenschaft finden sich außer bei Karl Eibl kaum Ausarbeitungen dieses Gedankens, obwohl literarischer Wandel häufiger als `Evolution` gedacht wird (vgl. die Angaben in Mellmann 2014, 95, Anm. 11) und der sogenannte >Literary Darwinism ‘ ein vieldiskutiertes Forschungsprogramm darstellt. ${ }^{33}$

Die brennenden Fragen für eine Theorie sozialer Selektion liegen auf der Hand: Was genau wird reproduziert (die Frage nach der Reproduktionseinheit), was genau wird selektiert bzw. ausgewählt ${ }^{34}$ (die Frage nach dem level of selection), nach welchen Kriterien wird ausgewählt und von wem (die Frage nach den Selektionsinstanzen)? Da man es im Fall der Entwicklung einer Textsorte mit mindestens vier verschiedenen Entitäten $\mathrm{zu}$ tun hat (der Textsorte selbst, der der Textsorte entsprechenden Textgruppe, textsortenspezifischen Produktionspraktiken, poetologisch-gattungsspezifischen Ideen), erfordert es einige Überlegung, um zu bestimmen, welche Entität repliziert und welche Entität ausgewählt wird: Will man die Analogie zur natürlichen Selektion aufrechterhalten, scheidet die Textsorte selbst als Replikationseinheit aus, da sie die ihm entsprechende Textgruppe nicht determiniert, wie das Gen seinen phänotypischen Effekt; die Relation zwischen Textsorte und Text ist eine von type und token, keine von Ursache und Wirkung. Bleiben also textsortenspezifische Produktionspraktiken und poetologisch-gattungsspezifische Ideen. Hier scheint es mir wiederum im Sinne einer Stärkung der Analogie zwischen natürlicher und sozialer Selektion ratsam, sich für die Praktiken als Replikationseinheit zu entscheiden, da sie die einer Textsorte entsprechende Textgruppe in stärkerem Maße determinieren als die poetologischen Ideen, die, wie in Abschnitt 6 gezeigt,

31 Einen einflussreichen terminologischen Vorschlag, mit der Einführung des Begriffs ımeme`, machte Richard Dawkins (vgl. 1976, Kap. 11). Auf seine Begriffsprägung geht das von 1997-2005 erschienene Journal of Memetics zurück.

32 Vgl. die Beiträge in der Sonderausgabe des Journal of Evolutionary Economy 16:6 (2006).

33 Vgl. etwa die Debatte in Scientific Study of Literature 3:1 (2013).

34 Aufgrund problematischer Konnotationen der Verben ıselektieren«", sselegieren « und sauslesen verwende ich an ihrer statt hier wie im Folgenden den weniger spezifischen Begriff rauswählen` für den Vorgang der Selektion. 
stark von der Praxis abweichen können. Repliziert werden also textsortenspezifische Produktionspraktiken, die zu einem unterschiedlich häufigen Auftreten von Textsorten führen.

Damit komme ich zur schwierigen Frage der Selektion und des Selektionsmechanismus. Klar scheint, welche These man verteidigen muss, um die Idee von sozialer Selektion literarischer Textsorten zu plausibilisieren: Ausgewählt werden die einer bestimmten Textsorte zugehörenden Texte - und damit indirekt die textsortenspezifischen Produktionspraktiken -, die eine Funktion für das Literatur- oder Gesellschaftssystem erfüllen. ${ }^{35}$ Selektionsinstanzen sind die Verlage, die Literaturkritik und in letzter Instanz das Publikum. Bei der Verteidigung dieser These treten allerdings zwei Schwierigkeiten auf, die zum einen auf Unterschieden zwischen sozialer und natürlicher Selektion und zum anderen auf Besonderheiten der Selektion auf dem literaturwissenschaftlichen Markt beruhen.

\section{Variation und /Vererbungs}

Erstens scheint mir eine Schwierigkeit darin zu bestehen, dass der Träger der Reproduktionseinheit (der Praktiken), d.i. der Schriftsteller, von der Entität unterschieden ist, an der sich die phänotypischen Effekte zeigen (die literarischen Werke). Im Gegensatz zu natürlicher Selektion, wo ein positives >Umwelturteil über einen Organismus automatisch die Reproduktion der entsprechenden Gene nach sich zieht, führt ein positives Urteil über das literarische Werk eines Schriftsteller keineswegs automatisch dazu, dass er weitere Werke derselben Textsorte produziert, oder, bei einem negativen Urteil, auf ihre Produktion verzichtet. Dass in den Rückkopplungsmechanismus zwischen Selektion und Reproduktion eine intentionale Bewertungsinstanz mit Freiheitsgraden eingebaut ist, scheint mir allerdings noch kein zwingendes Argument gegen die soziale Selektion literarischer Textsorten. Denn man könnte argumentieren, dass Schriftsteller, die ihre Werke ohne

35 Orientiert man sich bei der Untersuchung literarischen Wandels entgegen meiner Entscheidung in Abschnitt 2 statt an Textsorten an Genres als >literarisch-soziale Institutionen<, die Praktiken der Produktion und Regeln der Rezeption einschließen, so liegt das Problem noch komplizierter. Denn dann handelt es sich, mit einer Unterscheidung von Runciman, bei literarischem Wandel nicht mehr nur um »cultural evolution« von Praktiken, sondern um »social evolution « von Institutionen. Der entscheidende Unterschied besteht in der Art und Weise, wie neue `Meme zustande kommen. Im Fall der Praktiken werden sie durch Reinterpretation erzeugt, im Fall der Institutionen durch Aushandlungen von Rollen, die mit Verhaltenskonventionen verbunden sind $(2009,39)$. 
die Intention produzieren, Erfolg $\mathrm{zu}$ haben, eine Ausnahme sind, und dass rein quantitativ dennoch die Textsorten ausgewählt werden, die eine Funktion haben.

Teilweise wurde auch in der Möglichkeit der Akteure, der Selektion gewissermaßen zuvorzukommen bzw. sie zu vermeiden, ein Grund dafür gesehen, dass sich die Theorie der natürlichen Selektion nicht ohne Verlust der Erklärungskraft auf den sozialen bzw. kulturellen Bereich übertragen lässt (vgl. Cordes 2006, insbes. 537). Man wird dieser Position zugestehen müssen, dass soziale Selektion sich durch die Möglichkeit der "präventive[n] Autoselektion« erheblich von natürlicher Selektion unterscheidet (Eibl 1996, 24), ${ }^{36}$ einsichtig ist jedoch nicht, warum dies automatisch einen Verlust explanatorischer Kraft nach sich ziehen soll. Wenn Organismen willentlich ihren Genotyp anpassen könnten, um sich der Umwelt anzupassen, würde dies das Vorherrschen eines bestimmten Phänotyps in der Population nur beschleunigen. Das problematische Element besteht aus meiner Sicht nicht in der präventiven Anpassung, sondern in der Freiheit des einzelnen Akteurs, sich nicht anzupassen.

\section{Selektion}

Die zweite Schwierigkeit betrifft die Selektion selbst, bei der (jedenfalls im Fall der Verlage und der Literaturkritik) im Gegensatz zur natürlichen Selektion wiederum Intentionen eine wichtige Rolle spielen. Ein Paradebeispiel für soziale Selektion sind firmeninterne Entscheidungsheuristiken, die von Ökonomen der Chicago-School untersucht wurden (vgl. Elster 1994, 404; Wray 2002, 75). Diese argumentieren, dass effektiv profit-maximierende Entscheidungsheuristiken ausgewählt werden, weil Firmen mit nicht-profit-maximierenden Maximen in Konkurs gehen oder solche Heuristiken intern korrigiert werden. Es werden also genau die Heuristiken ausgewählt, die einen Nutzen für die Firmen haben: die profitmaximierenden. Im Unterschied zu diesem Beispiel scheint es mir jedoch nicht ausgemacht, dass der Literaturmarkt stets die Textsorten auswählt, die gesellschaftlichen Nutzen haben. Dies liegt nicht in erster Linie daran, dass es neben dem Publikum bzw. dem >Marktı als Selektionsinstanz noch die Verlage und die Literaturkritik als Selektionsinstanzen gibt, sondern daran, dass der `Code`, mit dem literarische Werke bewertet werden, nämlich, so man Gerhard Plumpe und Niels Werber folgt, langweilig/interessant (vgl. Plumpe/Werber 1993,

36 Es ist hier aus Platzgründen nicht möglich auf Eibls Verbindung zwischen Problemgeschichte und sozialer Selektion, nach der die »Problemsituation [...] als Selektionsinstanz« (1991, 361) fungiert, näher einzugehen. 
28-32), ${ }^{37}$ nicht mit der Dichotomie gesellschaftlich nützlich/nutzlos zusammenfällt. Wenigstens ein spätestens seit der klassischen Moderne wichtiges Kriterium ergibt sich nicht aus dem gesellschaftlichen >Nutzen<, sondern aus literatursysteminternen Entwicklungen: das Kriterium der literarischen Innovativität.

Auch dies scheint jedoch keine Schwierigkeit zu sein, die alle Träume von funktionalen Erklärungen literarischen Wandels mittels sozialer Selektion umgehend zerplatzen lässt. ${ }^{38}$ Denn man könnte zum einen versuchen zu argumentieren, dass solche Erklärungen auch dann valide sind, wenn gesellschaftlicher Nutzen nur eines von mehreren Selektionskriterien ist: Es kann sein, dass einzelne Werke einer bestimmten gesellschaftlich nützlichen Textsorte keinen Erfolg haben, weil sie als zu wenig innovativ empfunden werden, aber auf lange Sicht werde sich die fragliche Textsorte trotzdem durchsetzen, solange die Zugehörigkeit $\mathrm{zu}$ ihr die >Fitness des Werksı steigert, d.h. die Wahrscheinlichkeit, dass das Werk Erfolg hat. ${ }^{39}$ Zum anderen könnte man versuchen $\mathrm{zu}$ argumentieren, wie es wohl viele marxistische Literaturwissenschaftler tun würden, dass sich gewissermaßen hinter den ästhetischen Codes, mit denen Literaturkritiker und Verlage operieren, (ohne ihr Wissen) in Wirklichkeit doch ein Code wie gesellschaftlich nützlich/nutzlos verbirgt, nämlich die Unterscheidung zwischen Werken, die die bestehenden Herrschaftsverhältnisse legitimieren, und solchen, die es nicht tun. Hier wird deutlich, dass die Bewertung des Nutzeffekts im Sinne einer Sicherung des Fortbestands des Systems stark von normativen Hintergrundannahmen abhängt.

Neben der Frage, ob die Selektionsinstanzen des Literaturmarktes überhaupt nach Kriterien gesellschaftlicher Nützlichkeit verfahren, gibt es jedoch einen zweiten Punkt, der die Annahme sozialer Selektion für das Literatursystem problematisiert. Um eine funktionale Erklärung nach dem Schema Littles zu erhalten, müssten die Selektionsinstanzen nämlich (bewusst oder unbewusst) auch weitgehend zutreffend die gesellschaftlich nützlichen Textsorten bzw. Produktionspraktiken auswählen. Denn nur ein wirklicher, kein vermeintlicher benefit führt, bei Erfüllung der anderen Bedingungen, zu funktionalen Erklärungen.

Während ich bisher die generelle Annahme eines Mechanismus sozialer Selektion problematisiert habe, gibt es darüber hinaus Schwierigkeiten, die seine

37 Einen Überblick über alternative Vorschläge sowie Kritik am genannten Vorschlag findet sich in Berlemann 2011, 95-121.

38 Nicht immer sind solche jedoch der Anspruch von Vertretern von Theorien ssozialer Selektion< (vgl. Runciman 2009, 30 und 46).

39 Man könnte sogar weitergehen: Auf lange Sicht gewinne das Kriterium des gesellschaftlichen Nutzens an Gewicht, denn, da das Streben nach literarischer Innovativität nur die Entstehung bzw. die anfängliche Konjunktur einer Textsorte ıerklären` kann, deute ihre Konsolidierung auf einen gesellschaftlichen Nutzen hin. 
Annahme im Einzelfall betreffen. Erstens lassen sich oft mehrere Nutzeneffekte für ein- und dieselbe Textsorte postulieren, die mit unterschiedlichen ihrer Merkmale zusammenhängen. Hier stellt sich die Frage, »welches [ihre] strategischen Eigenschaften sind, die durch Selektion in Richtung auf Funktionalität geformt worden sind « (Stichweh 1999, 467). Zweitens ist kaum eine soziale Praktik frei von Nachteilen; Nutzen und Nachteil einer bestimmten Praktik lassen sich oft nicht leicht gegeneinander abwägen, sodass gar nicht klar ist, ob unter dem Strich überhaupt ein Nutzen besteht (vgl. Wray 2002, 77).

\section{Teleologische Erklärungsansätze}

Der Begriff teleologische Erklärung wird in der Fachliteratur (aus philosophiehistorischen Ursachen) nicht immer einheitlich verwendet. Teilweise werden auch funktionale oder ein bestimmter Typus der intentionalen Erklärungen unter die teleologischen subsumiert, weil sie sich einer Zweck-Mittel-Begrifflichkeit bedienen (vgl. Schmid 2011, 6f.). Es ist daher nützlich, zwei Begriffe teleologischer Erklärungen zu unterscheiden. Teleologische Erklärungen im weiteren Sinne sind alle, die mit der Annahme einer >Finalursache` (causa finalis) arbeiten, d. h. ein Geschehen durch sein Ziel oder seinen Zweck erklären. Teleologische Erklärungen im engeren Sinne zeichnen sich zusätzlich dadurch aus, dass sie weder mit der Annahme arbeiten, dass die causa finalis nützlich (funktionale Erklärung) ist, noch mit der Annahme, dass die causa finalis geplant bzw. intendiert ist (intentionale Erklärung).

Eine teleologische Erklärung im engeren Sinne für das Aufkommen der Fiktionalisierung von Reflexion schlägt der kalifornische Literaturwissenschaftler Thomas J. Harrison vor, wenn er behauptet, dass das fiktional-narrative Element in der Natur des Essays angelegt sei:

\footnotetext{
A conviction is the form of a decision already taken; an essay is rather the shape of the sinner life in the act of reaching a decision. [...] Not only does the essay give shape to a process preceding conviction, and perhaps deferring it forever. More important, it records the hermeneutical situation in which such decisions arise. For this reason the essay ultimately requires novelistic form, which can portray the living conditions in which thought is tangled. (1992, 4, Hervorhebung im Original; vgl. auch ebd., 17f.)
}

Die Gattung Essay hätte diesem essentialistischen Argument zufolge eine bestimmte Aufgabe oder einen Zweck, zu dessen Verwirklichung sie der »novelistic form«, d. h. des fiktional-narrativen Elementes bedarf. Die Entstehung des fiktionalen Essayismus wäre somit ein notwendiger Schritt zum Erreichen dieses immanenten Zwecks. Selbst wenn man von der hochgradigen wissenschaftstheoretischen Fragwürdigkeit solcher Erklärungen - jedenfalls wenn man sie als 
ätiologische Erklärungsversuche im Gegensatz zu lediglich gegenstandsbestimmenden Erklärungsversuchen (»die Gattung Essay ist eine solche, die zur ınovelistic form`strebt«) auffasst ${ }^{40}$ - absieht, hat der Erklärungsansatz eine große Schwäche. Er vermag nicht zu erklären, warum es im deutschen Sprachraum gerade im ersten Drittel des 20. Jahrhunderts zur Fiktionalisierung des Essays bzw. zur >Essayfizierung des Romans kommt. Die Annahme einer Natur des Essays, in der diese Entwicklung angelegt ist, reicht dafür allein nicht aus; die Erklärung ist offenbar zu unspezifisch.

\section{Strukturale Erklärungsansätze}

Obwohl sich Vorstellungen von der Literatur und den Wissenschaften gemeinsamen >Tiefenstrukturen in jüngeren Untersuchungen aus dem Umfeld der so genannten >Poetologien des Wissens` großer Beliebtheit erfreuen und, wie Olav Krämer gezeigt hat, häufig auch mit Erklärungsansprüchen verbunden werden, ${ }^{41}$ dürfte der Erklärungsbegriff, mit dem Literaturwissenschaftler heutzutage ad hoc wahrscheinlich am wenigsten anfangen können, der Begriff der strukturalen bzw. strukturellen Erklärung sein. Dieser war vor allem in der deutschen Literaturwissenschaft der 1970er Jahre gebräuchlich und ist noch heute im französischsprachigen Raum (l'explication structurale) verbreitet. ${ }^{42}$ Wie schon der Name vermuten lässt, stammt er aus dem Umfeld des Strukturalismus und ist mit der Dichotomie von Oberflächenphänomenen und Tiefenstrukturen verbunden. Eine strukturale Erklärung, für die es meines Wissens kein allgemein anerkanntes Schema gibt, führt die Oberflächenphänomene auf Beziehungen zwischen den Elementen der Tiefenstruktur zurück, etwa die statistische Dominanz der Kreuzkusinenheirat (die Heirat mit der Tochter des Mutterbruders) auf ihre besondere systematische Stellung (nah genug zum Aufbau langfristiger Bündnisse zwischen >Clans`, weit genug entfernt zur Wahrung des Inzesttabus) (vgl. Lévi-Strauss 1989, 88-106).

Einer der elaborierteren literaturwissenschaftlichen Ansätze zur Beschreibung von literarischem Wandel, der explizit Anspruch auf strukturale Erklärungen erhebt, ist die »strukturalistisch-genetische Methode« des französischen Literaturtheoretikers Lucien Goldmann. In Abgrenzung von einer »Inhaltssozio-

$40 \mathrm{Zu}$ dieser Unterscheidung vgl. Schmid 2011, 12f., der von einer »ätiologischen« und einer »konstitutiven Teleologiekonzeption« spricht.

41 Die Rede ist hier von den Ansätzen, die Krämer unter dem Label »Zirkulations-Untersuchungen« subsumiert (vgl. 2011, 98-113; zu den Erklärungsansprüchen insbes. 111f. und 105, Anm. 72). 42 Vgl. etwa Finke 1982, 104-106 und 202-208. Im französischen Sprachraum wird explication structuale oft als Gegenbegriff zu interprétation gebraucht (vgl. etwa Ricœur 1970, insbes. 188-194). 
logie«, also Versuchen einen Zusammenhang zwischen dem »Inhalt der literarischen Werke und dem Inhalt des Kollektivbewußtseins der sozialen Gruppen« herzustellen (Goldmann 1984a, 239), entwickelt dieser die These, dass die »Strukturen der imaginären Welt des Werkes mit den Denkstrukturen bestimmter sozialer Gruppen homolog sind oder wenigstens in einer intelligiblen Beziehung stehen, während der Schriftsteller, was den Inhalt der Werke betrifft [...], eine vollkommene Freiheit genießt« (ebd., 240). Besonderer Vorzug seiner Methode sei es »gleichzeitig verstehend und erklärend zu sein«:

Die einheitlich tragische Struktur der Pensées von Pascal und der Racineschen Tragödien herauszuarbeiten ist ein verstehender Vorgang; sie jedoch in den extremistischen Jansenismus einzuordnen, indem man dessen Struktur bloßlegt, ist ein Prozeß des Erklärens in Bezug auf die Schriften Pascals und Racines; die Einfügung des extremistischen Jansenismus in die Gesamtgeschichte des Jansenismus ermöglicht, den ersteren zu erklären und die zweite zu verstehen. (ebd., 246)

Strukturale Erklärungen nach Goldmann korrelieren also durch Interpretation offenzulegende Strukturen (bedeutender) literarischer Werke/Werkgruppen einerseits mit großteils unbewussten »Denkstrukturen« sozialer Gruppen andererseits oder Strukturen literarischer Gattungen mit Gesellschaftsstrukturen.

In der Essayismus-Forschung werden Strukturerklärungen meines Wissens nicht explizit vertreten. Allerdings lassen sich manche Thesen des in der jüngeren Forschung verbreiteten Ansatzes, Essayismus als Denk- und Schreibmodus bzw. als Denkhaltung zu begreifen, als Strukturerklärungen auffassen. Einer der bekanntesten dieser Ansätze im deutschen Sprachraum stammt von Wolfgang Müller-Funk, der Essayismus als »Modus des Erkennens« begreift, der in der Moderne »ubiquitär« wird (1995, 9 und 281):

Je länger die Moderne als ein von den Menschen tendenziell reflektiertes, entzaubertes Projekt andauert und sich gleichsam postmodern streckt, desto stärker dringt der essayistische Impuls in Philosophie und Literatur ein. [...] In der Selbstthematisierung der Moderne werden Philosophie und Literatur in ihrem Gestus essayistisch, sowohl programmatisch wie im Hinblick auf die Darstellungsform. (ebd., 280)

Essayismus als Tendenz der Gattungsentwicklung (und damit auch fiktionaler Essayismus) wird von Müller-Funk also in Beziehung gesetzt zu einer denkgeschichtlichen Tendenz. Dabei wird in einem ersten Schritt die Tiefenstruktur der Gattung Essay herausgearbeitet, es handelt sich um den Vorgang des »distilling from the genre a more ubiquitous [essayistic] spirit« (Wampole 2014, 2) (hier: Essayismus als »Gestus«). In einem zweiten Schritt identifiziert man denkgeschichtliche oder gesellschaftliche Tendenzen, die die dieser Struktur analog, Goldmann würde (mit einer Metapher) sagen »homolog«, sind (Essayismus als 
Reflexionsmodus). In einem dritten Schritt setzt man diese beiden Strukturen zueinander in Beziehung und erhält so eine Strukturerklärung. Interessanterweise sind Strukturerklärungen nicht streng gerichtet: Entweder der Essayismus »dringt « als »essayistischer Impuls in Philosophie und Literatur ein" und bringt die essayistischen »Darstellungsform[en]« hervor oder das Verhältnis ist genau umgekehrt, weil die »Gattung « Essay dem »Gestus [des Essayismus, B.G.] einst zum Durchbruch verhalf« (Müller-Funk 1995, 280f.).

Diese Bidirektionalität, die eine Unabhängigkeit von Kausalbeziehungen impliziert, findet sich auch bei Goldmann. Zwar bestimmt dieser die Denkstrukturen selbst als »Antwort[en] auf die Probleme [der sozialen Gruppe]« und versucht gelegentlich das Zustandekommen der Strukturhomologien zu plausibilisieren (1984a, 241), ausschlaggebend für die Gültigkeit der Erklärung scheint für ihn jedoch vor allem die »strenge Homologie« zwischen zwei Strukturen (ebd., 26). ${ }^{43}$ Demnach müsste es prinzipiell auch möglich sein die Gesellschaftsstruktur durch die Struktur einer literarischen Gattung zu erklären. Werden strukturale Erklärungen auf diese Weise als dezidiert nicht-kausal aufgefasst, stellt dies nach Meinung von Wissenschaftstheoretikern ihren explanatorischen Wert jedoch radikal infrage (vgl. Little 1991, 112).

\section{Zusammenfassung und Ausblick}

Ziel des Beitrags war es zu untersuchen, ob sich literarischer Wandel erklären lässt. Literarischer Wandel wurde dabei als sich zeitlich ändernde Instantiierung von literarischen Textsorten definiert. Diese möglicherweise etwas technisch anmutende Formulierung meint, dass ein- und dieselbe Textsortendefinition für Zeitabschnitt $t_{1}$ eine quantitativ und/oder qualitativ anders beschaffene Gruppe in ihm entstandener Texte herausgreift als für den Zeitabschnitt $t_{2}$. Am Beispiel der im ersten Drittel des 20. Jahrhunderts zu beobachtenden Konjunktur des fiktionalen Essayismus wurden denkbare oder in der Forschung bereits lancierte Erklärungsansätze, die verschiedene Erklärungstypen exemplifizieren, entfaltet. Für jeden Erklärungstyp wurden verschiedene theoretische und/oder anwendungsbezogene Schwierigkeiten herausgestellt.

43 Es ist nicht ohne Weiteres klar, ob mit dem der Biologie entlehnten, also metaphorisch gebrauchten Begriff `Homologie` mehr gemeint ist als `Ähnlichkeit`. An einer Stelle deutet Goldmann jedoch an, dass sich die beiden `homologen` Strukturen so ähnlich seien, »daß man von einer einzigen Struktur sprechen könnte, die sich auf zwei verschiedenen Ebenen ausdrückt.« (Goldmann 1984b, 28f.) Dies könnte die Homologie-Metapher, die ja selbst ein kausales Element (Abstammung von einem Merkmal) impliziert, erklären. 
Kausalerklärungen, die lange unangefochten als paradigmatischer Typus wissenschaftlicher Erklärungen galten, stehen in der Literaturgeschichte, solange keine Gesetzmäßigkeiten literarischen Wandels entdeckt werden, nicht zur Verfügung. Dennoch ist es im Zusammenspiel von biographischen und einflussgeschichtlichen Untersuchungen sowie statistischen Analysen prinzipiell möglich, kausale Faktoren literarischen Wandels zu bestimmen und entsprechende (Teil-)Erklärungen zu geben. Ein wissenschaftstheoretisch sehr gut analysierter Erklärungstyp, die intentionale Erklärung, ist in seiner Anwendung auf literarischen Wandel von Textreihen nicht nur sehr aufwändig, sondern in seiner Reichweite oft beschränkt. Zum einen bezieht er sich lediglich auf die Produktion eines Autors und muss daher iteriert angewendet werden, zum anderen ist er aufgrund der unzureichenden Spezifizität poetologischer Selbstkommentare selbst bei poetologisch sehr versierten Autoren häufig nur sehr eingeschränkt anwendbar.

Der funktionale Erklärungsansatz ist in Bezug auf die Reichweite vielversprechender, kämpft jedoch mit mehreren Schwierigkeiten. Die erste Schwierigkeit besteht darin, dass Literatur in den allerseltensten Fällen gesellschaftliche Probleme löst, der von funktionalen Erklärungen vorausgesetzte Nutzeffekt also in aller Regel nur darin bestehen kann, gesellschaftliche Probleme zu thematisieren bzw. zu reflektieren. Ein solcher Nutzeffekt setzt einen entsprechenden Bedarf zur Problemthematisierung voraus, der nicht aus dem Textkorpus, das erklärt werden soll, erschlossen, sondern unabhängig von diesem nachgewiesen werden sollte. Die zweite Schwierigkeit resultiert aus weitreichenden Annahmen, die nötig scheinen, um die Existenz einer zur natürlichen Selektion analogen sozialen Selektion für das Literatursystem zu plausibilisieren. Ohne diese Annahmen ist unklar, worin der für funktionale Erklärungen unabdingbare Feedback-Mechanismus besteht, der verantwortlich dafür ist, dass der gesellschaftliche Nutzeffekt von bestimmten Textsorten ihr Auftreten wahrscheinlicher macht. Diese Annahmen, die wohl nur als idealisierend bezeichnet werden können, lauten: (i) Der Literaturmarkt (d. h. Instanzen wie Verlage, Kritiker etc.) wählt Textsorten unter anderem und ungefähr einhellig nach ihrem gesellschaftlichen Nutzen aus; (ii) er wählt sie zutreffend aus (also solche, die tatsächlich einen gesellschaftlichen Nutzeffekt haben); (iii) Schriftsteller richten sich mit ihrer Produktion, bewusst oder unbewusst, nach den Erfolgschancen bestimmter Textsorten auf dem literarischen Markt. Es wäre jedoch lohnend zu prüfen, unter welchen Umständen ein solches Set von Annahmen, sich als Teil einer legitimen, komplexitätsreduzierenden Modellbildung begreifen lässt.

Während der funktionale Erklärungsansatz auf weitreichende, idealisierende Hintergrundannahmen angewiesen ist, sind teleologische Erklärungen, die bestimmten Genres in ihnen angelegte Entwicklungstendenzen zuschreiben, nicht nur wissenschaftstheoretisch dubios, sondern auch $\mathrm{zu}$ unspezifisch, weil sie 
nicht erklären können, warum sich diese Tendenzen erst in einer spezifischen historischen Situation manifestieren. Ebenfalls fragwürdig sind strukturale Erklärungen, die literarische `Tiefenstrukturen identifizieren, die als `homolog` bzw. analog zu sozialen oder ideengeschichtlichen Strukturen begriffen werden. Die Fragwürdigkeit solcher Erklärungen beruht auf der Tatsache, dass sie keine (wirkliche) Auskunft über die Mechanismen geben (können), die für das Zustandekommen der Strukturanalogien verantwortlich sind, ihr explanatorischer Wert jedoch genau an diesen Mechanismen zu hängen scheint.

Insbesondere die strukturalen Erklärungen legen einen Gedanken nahe, der gewissermaßen an den Anfang des Aufsatzes zurückführt: Ließen sich strukturale Erklärungen nicht viel besser so beschreiben, dass sie ein Verstehen herbeiführen, indem sie einen Zusammenhang zwischen analogen Phänomenen, etwa zwischen Essayismus bzw. fiktionalem Essayismus als Tendenz der Gattungsentwicklung und Essayismus als modernem Reflexionsmodus, herstellen? Diese Frage ließe sich auf die anderen Erklärungstypen ausweiten. Möglicherweise leisten kausale, funktionale, teleologische oder strukturale Erklärungsansätze in der Literaturwissenschaft etwas, das auch literaturwissenschaftlichen Wert hat, obwohl sie keine vollwertigen bzw. vollgültigen Erklärungen darstellen. Der Mehrwert dieser, nennen wir sie behelfsweise, übergeordneten Narrative gegenüber der Beschreibung lässt sich präsumtiv mit lerntheoretischen bzw. informationstheoretischen Begriffen wie 'Wissensverknüpfung`, 'Wissenssynthese` und `Komplexitätsreduktion` erläutern, was hier nur angedeutet werden kann. ${ }^{44}$ Weniger geeignet zu diesem Zweck ist hingegen der traditionsreiche Begriff , Verstehen «, der unter anderem das Ergebnis von Beschreibungen, Interpretationen und Erklärungen bezeichnen kann. Ein Erläuterung des Mehrwerts der übergeordneten Narrative mithilfe der Dichotomie Verstehen/Erklären schiene mir die bis hierhin erreichten Differenzierungsgewinne daher eher wieder zunichtezumachen.

Wichtiger als dies in extenso zu diskutieren, scheint mir die abschließende Frage, welchen Schluss man aus dem Befund ziehen soll, dass vollwertige Erklärungen literarischen Wandels nur mit sehr großem Aufwand (statistische und intentionale Erklärungen) oder unter idealisierenden Hintergrundannahmen (funktionale Erklärungen) zu haben sind. Welche Einstellung soll der wissenschaftstheoretisch interessierte Literaturwissenschaftler gegenüber den unzähligen existierenden übergeordneten Narrativen und solchen, die sich ihm in seiner eigenen Forschung aufdrängen, einnehmen, wenn sie präsumtiv als Scheinerklärungen,

44 So scheint etwa ein kaum gewürdigter Vorzug des problemgeschichtlichen Ansatzes darin zu bestehen, dass die Problem-Lösungs-Figur es erlaubt, einer Textreihe begründet einen gemeinsamen Kontext zuzuweisen und so vergleichbare Interpretationen zu generieren. 
wenn auch mit Mehrwert, zu qualifizieren sind? Grundsätzlich bieten sich hier zwei Optionen an, die entweder die Diskontinuität oder die Kontinuität literaturwissenschaftlicher Praxis favorisieren: (a) der Verzicht auf den Anspruch literarischen Wandel zu erklären, d. h. die Revision der bestehenden Praxis oder (b) die Weiterführung der bestehenden Praxis unter ihrer Neubeschreibung.

Die erste Option hätte zwei vergleichsweise radikale Implikationen. Zum einen müssten die existierenden übergeordneten Narrative aus dem Kanon des zu tradierenden Wissens ausgeschieden werden. Zum anderen müsste prospektiv in der Forschung darauf verzichtet werden, Warum-Fragen in Bezug auf literarischen Wandel zu beantworten. Die zweite Option scheint demgegenüber attraktiver, weil sie die Fortführung der Praxis, d. h. der Tradierung und Generierung übergeordneter Narrative, erlaubt, die Warum-Fragen in Bezug auf literarischen Wandel beantworten. Die Option beinhaltet jedoch eine Neubeschreibung dieser Praxis, die selbst eine Herausforderung darstellt. Da den übergeordneten Narrativen per definitionem keine vollwertige Erklärung entspricht, müsste erstens auf den Erklärungsbegriff im Zusammenhang mit ihnen verzichtet werden. An alternativen schwächeren Ausdrücken wie >Darstellung historischer Zusammenhänge` oder `Erläuterung von Bedingungsverhältnissen` mangelt es nicht. Zweitens, und dies ist der schwierige Teil, müsste ein Kriterium zur Unterscheidung von übergeordneten Narrativen und Scheinerklärungen entwickelt werden. Denn mit Evidenz taucht hier das in Abschnitt 3 formulierte Kriterienproblem für literaturwissenschaftliche Erklärungen in gewandelter Form wieder auf: Ein übergeordnetes Narrativ ist in einem unbestimmten Sinne >mehr` als Beschreibung, aber weniger als eine vollwertige Erklärung. Doch wie genau lässt sich dieses >Mehr abgrenzen, wenn nicht jede Operation, die eine Textreihe mit einem Kontext verknüpft als übergeordnetes Narrativ gelten soll? Mit dieser Frage steht man offenkundig vor einer ähnlich schwer lösbaren Aufgabe wie jener, allgemeine Kriterien für literaturwissenschaftliche Erklärungen $\mathrm{zu}$ entwickeln. ${ }^{45}$ Möglicherweise wäre hier jedoch eine praxeologische Untersuchung literaturgeschichtlicher Darstellungen (analog zur jüngst projektierten Untersuchung von Interpretationen, vgl. Albrecht et al. 2015) ein entscheidender Schritt, denn präsumtiv sind übergeordnete Narrative genau das, was gute Literaturwissenschaftler in der Praxis immer schon produzieren, wenn sie literarischen Wandel darstellen.

45 Anhaltspunkte finden sich in der Debatte um sogenannte snarrative Erklärungen in der Geschichtswissenschaft, obwohl dort meistens akteursfokussierte Explananda im Mittelpunkt stehen. Für einen Überblick vgl. Frings 2008; zu den Möglichkeiten einer Anwendung in der Literaturwissenschaft insbes. 163, Anm. 87. 
Aus dieser Perspektive ließe sich auch der vielleicht etwas verdrießlich stimmenden Zusammenfassung ein optimistischer Schluss abgewinnen: Schlecht steht es nicht notwendigerweise um die literaturwissenschaftliche Praxis der `Erklärung ‘ literarischen Wandels, sondern um ihre Beschreibung und das Selbstverständnis des historisch arbeitenden Literaturwissenschaftlers, zu deren Erneuerung dieser Aufsatz beitragen möchte.

Danksagung: Die Entstehung dieses Aufsatzes wurde ermöglicht durch die großzügige Förderung der Fritz Thyssen Stiftung. Für wertvolle Hinweise und Kritik danke ich Olav Krämer (Freiburg), Dirk Werle (Heidelberg) sowie der Herausgeberin und den Herausgebern des Journal of Literary Theory.

\section{Literatur}

Achinstein, Peter, The Nature of Explanation, New York 1983.

Ajouri, Philip, Erzählen nach Darwin. Die Krise der Teleologie im literarischen Realismus. Friedrich Theodor Vischer und Gottfried Keller, Berlin/ New York 2007.

Albrecht, Andrea et al. (Hg.), Theorien, Methoden und Praktiken des Interpretierens, Berlin et al. 2015.

Aristoteles, Metaphysik. Griechisch-deutsch [1978], Neub. d. Übersetzung von Hermann Bonitz, mit Einleitung und Kommentar hrsg. von Horst Seidl, 2 Bde., Hamburg ${ }^{3}$ 1989/1991.

Beckermann, Ansgar, Gründe und Ursachen, Kronberg i.Ts. 1977.

Berlemann, Dominic, Wertvolle Werke. Reputation im Literatursystem, Bielefeld 2011.

Bollnow, Otto Friedrich, Dilthey. Eine Einführung in seine Philosophie [1936], Stuttgart et al. ${ }^{3} 1967$.

Borkowski, Jan, Literatur und Kontext. Untersuchungen zum Text-Kontext-Problem aus textwissenschaftlicher Sicht, Münster 2015.

Borkowski, Jan/Philipp David Heine, Ziele der Literaturgeschichtsschreibung, Journal of Literary Theory 7:1-2 (2013), 31-63.

Brigandt, Ingo, Explanation in Biology. Reduction, Pluralism, and Explanatory Aims, Science \& Education 20:1 (2013), 69-91.

Broch, Hermann, Gesammelte Werke, Bd. 8: Briefe von 1929 bis 1951, hg. von Robert Pick, Zürich 1957.

Bühler, Axel, Interpretieren - Vielfalt oder Einheit?, in: Fotis Jannidis et al. (Hg.), Regeln der Bedeutung. Zur Theorie der Bedeutung literarischer Texte, Berlin/New York 2003, 169-181.

Burdorf, Dieter, Gespräche über Kunst. Zur Konjunktur einer literarischen Form um 1900, in: Andreas Beyer/Dieter Burdorf (Hg.), Jugendstil und Kulturkritik. Zur Literatur und Kunst um 1900, Heidelberg 1999, 29-50.

Buschmeier, Matthias, Literaturgeschichte nach dem Ende der Theorie? Thesen zu den (Un-) Möglichkeiten einer bedrohten Gattung, Internationales Archiv für Sozialgeschichte der deutschen Literatur 36:2 (2011), 409-414. 
Buschmeier, Matthias/Walter Erhart/Kai Kauffmann, Einleitung, in: M.B./W.E./K.K. (Hg.), Literaturgeschichte. Theorien - Modelle - Praktiken, Berlin 2014, 1-7.

Carlshamre, Staffan/Anders Pettersson, Types of Interpretation in the Aesthetic Disciplines, Montréal 2003.

Cartwright, Nancy, From Causation to Explanation and Back, in: Brian Leiter (Hg.), The Future for Philosophy, Oxford/New York 2004, 230-245.

Cordes, Christian, Darwinism in Economics. From Analogy to Continuity, Journal of Evolutionary Economics 16 (2006), 529-541.

Danneberg, Lutz, Quelle, Einfluss, 2008, http://www.fheh.org/index.php/projekte/analysekategorien/38-analysekategorien/115-quelle-einfluss (26.09.2014).

Dawkins, Richard, The Selfish Gene, Oxford 1976.

Detel, Wolfgang, Hermeneutik und Erklärung, Mythos-Magazin (2006), http://www.mythosmagazin.de/erklaerendehermeneutik/wd_erklaerung.pdf. (31.05.2016).

Dittrich, Andreas, Glauben, Wissen und Sagen. Studien zu Wissen und Wissenskritik im »Zauberberg", in den »Schlafwandlern« und im »Mann ohne Eigenschaften«, Tübingen 2009.

Eibl, Karl, Zurück zu Darwin. Bausteine zur historischen Funktionsbestimmung von Dichtung, in: Michael Titzmann (Hg.), Modelle des literarischen Strukturwandels, Tübingen 1991, 347366.

Eibl, Karl, Die Entstehung der Poesie, Frankfurt a. M. 1995.

Eibl, Karl, Literaturgeschichte, Ideengeschichte, Gesellschaftsgeschichte - und >Das Warum der Entwicklung`, Internationales Archiv für Sozialgeschichte der deutschen Literatur 21:2 (1996), 1-26.

Eibl, Karl, Autonomie und Funktion, Autopoiesis und Kopplung. Ein Erklärungsangebot für ein literaturwissenschaftliches Methodenproblem mit einem Blick auf ein fachpolitisches Problem, in: Martin Huber/Gerhard Lauer (Hg.), Nach der Sozialgeschichte. Konzepte für eine Literaturwissenschaft zwischen Historischer Anthropologie, Kulturgeschichte und Medientheorie, Tübingen 2000, 175-190.

Elster, Jon, Functional Explanation: In Social Science, in: Michael Martin/Lee C. Mclntyre (Hg.), Readings in the Philosophy of Social Science, Cambridge, MA 1994, 403-414.

Elster, Jon, Explaining Social Behavior. More Nuts and Bolts for the Social Sciences, Cambridge 2007.

Fanta, Walter, Die Entstehungsgeschichte des »Mann ohne Eigenschaften« von Robert Musil, Wien et al. 2000.

Finke, Peter, Konstruktiver Funktionalismus. Eine wissenschaftstheoretische Basis einer empirischen Theorie der Literatur, Braunschweig/Wiesbaden 1982.

Fluck, Winfried, Funktionsgeschichte und ästhetische Erfahrung, in: Marion Gymnich/Ansgar Nünning (Hg.), Funktionen von Literatur. Theoretische Grundlagen und Modellinterpretationen, Trier 2005, 29-53.

Fowler, Alastair, The Life and Death of Literary Forms, New Literary History 2:2 (1971), 199-216.

Fraassen, Bas C. van, The Scientific Image, Oxford 1980.

Fricke, Harald, Norm und Abweichung. Eine Philosophie der Literatur, München 1981.

Fricke, Harald, Invarianz und Variabilität von Gattungen, in: Rüdiger Zymner (Hg.), Handbuch Gattungstheorie, Stuttgart 2010, 19-21.

Frings, Andreas, Erklären und Erzählen. Narrative Erklärungen historischer Sachverhalte, in: A.F./Johannes Marx (Hg.), Erzählen, Erklären, Verstehen. Beiträge zur Wissenschaftstheorie und Methodologie der historischen Kulturwissenschaften, Berlin 2008, 129-164. 
Frisé, Adolf, Roman und Essay. Gedanken u. a. zu Hermann Broch, Thomas Mann und Robert Musil, in: A.F., Plädoyer für Robert Musil. Hinweise und Essays 1931 bis 1980 [1960], Reinbek b.H. 1980, 80-99.

Fulda, Daniel, Starke und schwache Historisierung im wissenschaftlichen Umgang mit Literatur. Zur Frage, was heute noch möglich ist - mit einer disziplingeschichtlichen Rückblende, in: Matthias Buschmeier/Walter Erhart/Kai Kauffmann (Hg.), Literaturgeschichte. Theorien Modelle - Praktiken, Berlin 2014, 101-121.

Gill, Jameson, Memes and Narrative Analysis. A Potential Direction for the Development of NeoDarwinian Orientated Research in Organisations, Euram. Proceedings of the European Academy of Management 11 (2011), http://shura.shu.ac.uk/4241/1/Memes_and_Narrative \%5B1\%5D.pdf (21.06.2016).

Gittel, Benjamin, Lebendige Erkenntnis und ihre literarische Kommunikation. Robert Musil im Kontext der Lebensphilosophie, Münster 2013.

Gittel, Benjamin, Die Bestätigung von Interpretationshypothesen zu fiktionalen literarischen Werken, in: Andrea Albrecht et al. (Hg.), Theorien, Methoden und Praktiken des Interpretierens, Berlin et al.2015, 513-564 (Gittel 2015a).

Gittel, Benjamin, Essayismus als Fiktionalisierung von unsicheres Wissen prozessierender Reflexion, Scientia Poetica 19 (2015), 136-171 (Gittel 2015b).

Gittel, Benjamin, Niemals aber sagt ein lebendiger Mensch zu einem anderen ... ıSei mein Erlöser! ‘. Drei Arten der Fiktionalisierung von weltanschaulicher Reflexion bei Broch, Lukács und Musil, Zeitschrift für deutsche Philologie 135:2 (2016), 213-244.

Godfrey-Smith, Peter, Theory and Reality. An Introduction to the Philosophy of Science, Chicago et al. 2009.

Goldmann, Lucien, Die strukturalistisch-genetische Methode in der Literaturwissenschaft, L.G., Soziologie des Romans, übers. von L.G. und Ingeborg Fleischhauer, Frankfurt a. M. 1984, 233-256 (Goldman 1984a).

Goldmann, Lucien, Einführung in die Probleme einer Soziologie des Romans, L.G., Soziologie des Romans, übers. von L.G. und Ingeborg Fleischhauer, Frankfurt a. M. 1984, 17-40 (Goldman 1984b).

Greimas, Algirdas Julien, Sur l'histoire événementielle et l'histoire fondamentale, in: Reinhart Koselleck/Wolf-Dieter Stempel (Hg.), Geschichte, Ereignis und Erzählung, München 1973, 139-153.

Gymnich, Marion, Gattung und Gattungshistoriographie, in: Rüdiger Zymner (Hg.), Handbuch Gattungstheorie, Stuttgart 2010, 131-158.

Gymnich, Marion/Ansgar Nünning, Funktionsgeschichtliche Ansätze. Terminologische Grundlagen und Funktionsbestimmungen von Literatur, in: M.G./A.N. (Hg.), Funktionen von Literatur. Theoretische Grundlagen und Modellinterpretationen, Trier 2005, 3-28.

Harrison, Thomas J., Essayism. Conrad, Musil \& Pirandello, Baltimore 1992.

Hedström, Peter/Petri Ylikoski, Causal Mechanisms in the Social Sciences, Annual Review of Sociology 36 (2010), 49-67.

Henninger, Peter, Der Buchstabe und der Geist. Unbewußte Determinierung im Schreiben Robert Musils, Frankfurt a. M. et al. 1980.

Hermerén, Göran, Interpretation. Types and Criteria, Grazer philosophische Studien 19 (1983), 131-161.

Hesse, Hermann, Das Glasperlenspiel. Versuch einer allgemeinverständlichen Einführung in seine Geschichte, Neue Rundschau 12 (1934), 638-665. 
Iser, Wolfgang, A German View of Walter Pater, in: Stephen Bann (Hg.), The Reception of Walter Pater in Europe, London 2004, 142-151.

Jander, Simon, Die Poetisierung des Essays. Rudolf Kassner, Hugo von Hofmannsthal, Gottfried Benn, Heidelberg 2008.

Jannidis, Fotis, Literarisches Wissen und Cultural Studies, in: Martin Huber/Gerhard Lauer (Hg.), Nach der Sozialgeschichte, Tübingen 2000, 335-358.

Kiesel, Helmuth, Geschichte der literarischen Moderne. Sprache, Ästhetik, Dichtung im zwanzigsten Jahrhundert, München 2004.

Kincaid, Harold, Contextualism, Explanation and the Social Sciences, Philosophical Explorations 7:3 (2004), 201-218.

Kindt, Tom/Hans-Harald Müller, Wieviel Interpretation enthalten Beschreibungen? Überlegungen zu einer umstrittenen Unterscheidung am Beispiel der Narratologie, in: Fotis Jannidis et al. (Hg.), Regeln der Bedeutung. Zur Theorie der Bedeutung literarischer Texte, Berlin/New York 2003, 286-304.

King, Martina/Jesko Reiling, Das Text-Kontext-Problem in der literaturwissenschaftlichen Praxis: Zugänge und Perspektiven, Journal of Literary Theory 8:1 (2014), 2-30.

Klausnitzer, Ralf, Koexistenz und Konkurrenz. Theoretische Umgangsformen mit Literatur im Widerstreit, in: R.K./Carlos Spoerhase (Hg.), Kontroversen in der Literaturtheorie/Literaturtheorie in der Kontroverse, Berlin et al. 2007, 15-48.

Koertge, Noretta, Explanation and its Problems, British Journal of Philosophy of Science 43 (1992), 85-98.

Krämer, Olav, Intention, Korrelation, Zirkulation. Zu verschiedenen Konzeptionen der Beziehung zwischen Literatur, Wissenschaft und Wissen, in: Tilmann Köppe (Hg.), Literatur und Wissen - Sondierung eines Forschungsterrains, Berlin/New York 2011, 77-115.

Kreutzer, Leo, Erkenntnistheorie und Prophetie. Hermann Brochs Romantrilogie "Die Schlafwandler«, Tübingen 1966.

Lévi-Strauss, Claude, Die elementaren Strukturen der Verwandtschaft [1981], übers. von Eva Moldenhauer, Frankfurt a. M. ${ }^{2} 1984$.

Little, Daniel, Varieties of Social Explanation. An Introduction to the Philosophy of Social Science, San Francisco/Oxford 1991.

Luhmann, Niklas, Verfassung als evolutionäre Errungenschaft, Rechtshistorisches Journal 9 (1990), 176-220.

Luhmann, Niklas, Die Gesellschaft der Gesellschaft [1997], Frankfurt a. M. ${ }^{3} 2001$.

Lukács, Georg, Die Seele und die Formen. Essays, Berlin 1911.

Mellmann, Katja, Kontext ıGesellschaftı. Literarische Kommunikation-Semantik-Strukturgeschichte, Journal of Literary Theory 8:1 (2014), 87-117.

Menges, Karl, Kritische Studien zur Wertphilosophie Hermann Brochs, Tübingen 1970.

Mitchell, Sandra, Complexity and Explanation in the Social Sciences, in: Chrysostomos Mantzavinos (Hg.), Philosophy of the Social Sciences. Philosophical Theory and Scientific Practice, Cambridge 2009, 130-145.

Moser, Walter, Diskursexperimente im Romantext. Zu Musils `Mann ohne Eigenschaften`, in: Uwe Baur/Elisabeth Castex (Hg.), Robert Musil. Untersuchungen, Königstein i.Ts. 1980, 170-198.

Müller, Dorrit, >Lufthiebe streitbarer Privatdocentenヶ. Kontroversen um die theoretische Grundlegung der Literaturwissenschaft (1890-1910), in: Ralf Klausnitzer/Carlos Spoerhase (Hg.), Kontroversen in der Literaturtheorie/Literaturtheorie in der Kontroverse, Berlin et al. 2007, 149-170. 
Müller, Jan-Dirk, Literaturgeschichte/Literaturgeschichtsschreibung, in: Dietrich Harth/Peter Gebhardt (Hg.), Erkenntnis der Literatur. Theorien, Konzepte, Methoden der Literaturwissenschaft, Stuttgart 1982, 195-227.

Müller-Funk, Wolfgang, Erfahrung und Experiment. Studien zu Theorie und Geschichte des Essayismus, Berlin 1995.

Musil, Robert, Klagenfurter Ausgabe. Kommentierte digitale Edition sämtlicher Werke, Briefe und nachgelassener Schriften. Mit Transkriptionen und Faksimiles aller Handschriften. Lesetexte, Bd. 16: Frühe Tagebuchhefte (1899-1926), hg. von Walter Fanta/Klaus Amann/Karl Corino (Musil 2009a).

Musil, Robert, Erzählungstechnik, in: R.M., Klagenfurter Ausgabe. Kommentierte digitale Edition sämtlicher Werke, Briefe und nachgelassener Schriften. Mit Transkriptionen und Faksimiles aller Handschriften. Lesetexte, Bd. 14: Gedichte, Aphorismen und Selbstkommentare/ Selbstkommentare aus dem Nachlass/ Zum Roman. Parerga und Paralipomena 1921-1937, hg. von Walter Fanta/Klaus Amann/Karl Corino, Mappe II/1/142-147 (Musil 2009b).

Musil, Robert, Geist und Erfahrung. Anmerkungen für Leser, welche dem Untergang des Abendlandes entronnen sind, in: R.M., Klagenfurter Ausgabe. Kommentierte digitale Edition sämtlicher Werke, Briefe und nachgelassener Schriften. Mit Transkriptionen und Faksimiles aller Handschriften. Lesetexte, Bd. 12: Essays/ 1918-1926, hg. von Walter Fanta/Klaus Amann/Karl Corino, 841-858 (Musil 2009c).

Nünning, Ansgar, Semantisierung literarischer Formen, in: A.N. (Hg.), Metzler Lexikon Literaturund Kulturtheorie. Ansätze - Personen - Grundbegriffe [1998], Stuttgart/Weimar ${ }^{4} 2008$, $579 f$.

Ort, Claus-Michael, Das Wissen der Literatur. Probleme einer Wissenssoziologie literarischer Semantik, in: Tilmann Köppe (Hg.), Literatur und Wissen. Sondierung eines Forschungsterrains, Berlin/New York 2011, 164-191.

Ort, Claus-Michael/Friedericke Meyer, Konzept eines struktural-funktionalen Theoriemodells für eine Sozialgeschichte der Literatur, in: Renate von Heydebrand/Dieter Pfau/Jörg Schönert (Hg.), Zur theoretischen Grundlegung einer Sozialgeschichte der Literatur. Ein strukturalfunktionaler Entwurf, Tübingen 1988, 85-171.

Plumpe, Gerhard/Niels Werber, Literatur ist codierbar, in: Siegried J. Schmidt (Hg.), Literaturwissenschaft und Systemtheorie. Positionen, Kontroversen, Perspektiven, Opladen 1993, 9-34.

Ricœur, Paul, Qu'est-ce qu'un Texte? Expliquer et Comprendre, in: Rüdiger Bubner/Konrad Cramer/Reiner Wiehl (Hg.), Hermeneutik und Dialektik, Bd. 2: Sprache und Logik. Theorie und Auslegung und Probleme der Einzelwissenschaften, Tübingen 1970, 181-200.

Runciman, Walter Garrison, The Theory of Cultural and Social Selection, Cambridge, MA 2009.

Salmon, Wesley C., Four Decades of Scientific Explanation, Minneapolis 1989.

Salmon, Wesley C., Causality and Explanation, New York/Oxford 1998 (Salmon 1998a).

Salmon, Wesley C., Causality in Archaeological Explanations, in: W.C.S., Causality and Explanation, New York/Oxford 1998, 347-355 (Salmon 1998b).

Salmon, Wesley C., Comets, Pollen and Dreams. Some Reflections on Scientific Explanation, in: W.C.S., Causality and Explanation, New York/Oxford 1998, 50-67 (Salmon 1998c).

Salmon, Wesley C., Why Ask 'Whyı? An Inquiry Concerning Scientific Explanation, in: W.C.S., Causality and Explanation, New York/Oxford 1998, 125-141 (Salmon 1998d).

Salmon, Wesley C./Merrilee H. Salmon, Alternative Models of Scientific Explanation, in: W.C.S., Causality and Explanation, New York/Oxford 1998, 333-346.

Schlaffer, Hannelore, Der kulturkonservative Essay des 20. Jahrhunderts, in: Heinz Schlaffer/H. S. (Hg.), Studien zum ästhetischen Historismus, Frankfurt a. M. 1975, 140-173. 
Schmid, Stephan, Finalursachen in der frühen Neuzeit. Eine Untersuchung der Transformation teleologischer Erklärungen, Berlin/New York 2011.

Schönert, Jörg, Einleitung. Möglichkeiten und Probleme von Literaturgeschichte in Gesellschaftsund Kulturgeschichte, in: Lutz Danneberg/Jörg Schönert (Hg.), Wissen in Literatur im 19. Jahrhundert, Tübingen 2002, 337-348.

Schützeichel, Rainer, Sinn als Grundbegriff bei Niklas Luhmann, Frankfurt a. M./New York 2003.

Seeber, Hans Ulrich, Funktionen der Literatur im Prozess der Modernisierung, in: Marion Gymnich/Ansgar Nünning (Hg.), Funktionen von Literatur. Theoretische Grundlagen und Modellinterpretationen, Trier 2005, 79-97.

Sloterdijk, Peter, Weltanschauungsessayistik und Zeitdiagnostik, in: Bernhard Weyergraf (Hg.), Hansers Sozialgeschichte der deutschen Literatur vom 16. Jahrhundert bis zur Gegenwart, Bd. 8: Literatur der Weimarer Republik 1918-1933, München/Wien 1995, 309-339.

Sommer, Roy, Funktionsgeschichten. Überlegungen zum Funktionsbegriff in der Literaturwissenschaft und Anregungen zu seiner Differenzierung, Literaturwissenschaftliches Jahrbuch 41 (2000), 319-341.

Spoerhase, Carlos, Autorschaft und Interpretation. Methodische Grundlagen einer philologischen Hermeneutik, Berlin/New York 2007.

Steel, Daniel, Mechanisms and Functional Hypotheses in Social Sciences, Philosophy of Science 72 (2005), 941-952.

Stichweh, Rudolf, Kultur, Wissen und die Theorien soziokultureller Evolution, Soziale Welt 50:4 (1999), 459-470.

Striedter, Jurij, Literary Structure, Evolution, and Value. Russian Formalism and Czech Structuralism Reconsidered, Cambridge, MA et al. 1989.

Titzmann, Michael, Skizze einer integrativen Literaturgeschichte, in: M.T. (Hg.), Modelle des literarischen Strukturwandels, Tübingen 1991, 395-438.

Titzmann, Michael, >Problem - Problemlösungı als literarhistorisches und denkgeschichtliches Interpretationsinstrument, Scientia Poetica 14 (2010), 298-332.

Tucholsky, Kurt [u.d.N. Peter Panter], Rezept des Feuilletonisten, Die Weltbühne 35 (31.08.1922), 237.

Vanderbeeken, Robrecht, Models of Intentional Explanation, Philosophical Explorations 7:3 (2004), 233-246.

Vollhardt, Friedrich, Hermann Brochs geschichtliche Stellung. Studien zum philosophischen Frühwerk und zur Romantrilogie »Die Schlafwandler« (1914-1932), Tübingen 1986.

Voßkamp, Wilhelm, Gattungen als literarisch-soziale Institutionen, in: Walter Hinck (Hg.), Textsortenlehre, Gattungsgeschichte, Heidelberg 1977.

Voßkamp, Wilhelm, Zur Konstituierung eines gattungsgeschichtlichen Prototyps, in: W.V. (Hg.), Utopieforschung. Interdisziplinäre Studien zur neuzeitlichen Utopie, Stuttgart 1982, 183196.

Wampole, Christy Nicole, Late Twentieth-century French and Italian Essayistic Fiction, PhD-Thesis Stanford, http://udini.proquest.com/view/late-twentieth-century-frenchand-pqid:2438690401/ (07.04.2014).

Werle, Dirk, Frage und Antwort, Problem und Lösung. Zweigliedrige Rekonstruktionskonzepte literaturwissenschaftlicher Ideenhistoriographie, Scientia Poetica 17 (2009), 255-303.

Werle, Dirk, Problem und Kontext. Zur Methodologie der literaturwissenschaftlichen Problemgeschichte, Journal of Literary Theory 8:1 (2014), 31-54.

Wolf, Norbert Christian, Kakanien als Gesellschaftskonstruktion. Robert Musils Sozioanalyse des 20. Jahrhunderts, Wien 2011. 
Woodward, James, Making Things Happen. A Theory of Causal Explanation, New York/Oxford 2003.

Wray, Brad K., Social Selection, Agent's Intentions, and Functional Explanations, Analyse \& Kritik 24 (2002), 72-86.

Žmegač, Victor, Einleitung, in: V.ž. (Hg.), Geschichte der deutschen Literatur vom 18. Jahrhundert bis zur Gegenwart [1978], Bd. I:1, Weinheim 41996, IX-XXXI. 